\title{
Logic programs with monotone abstract constraint atoms*
}

\author{
Victor W. Marek \\ Department of Computer Science, University of Kentucky, Lexington, KY 40506, USA \\ (e-mail: marek@cs.uky.edu) \\ Ilkka Niemelä \\ Department of Computer Science and Engineering \\ Helsinki University of Technology, \\ P.O.Box 5400, FI-02015 TKK, Finland \\ (e-mail: Ilkka.Niemela@tkk.fi) \\ Mirosław Truszczyński \\ Department of Computer Science, University of Kentucky, Lexington, KY 40506, USA \\ (e-mail: mirek@cs.uky.edu) \\ submitted 30 June 2004; revised 7 February 2006; accepted 23 August 2006
}

\begin{abstract}
We introduce and study logic programs whose clauses are built out of monotone constraint atoms. We show that the operational concept of the one-step provability operator generalizes to programs with monotone constraint atoms, but the generalization involves nondeterminism. Our main results demonstrate that our formalism is a common generalization of (1) normal logic programming with its semantics of models, supported models and stable models, (2) logic programming with weight atoms (lparse programs) with the semantics of stable models, as defined by Niemelä, Simons and Soininen, and (3) of disjunctive logic programming with the possible-model semantics of Sakama and Inoue.

To appear in Theory and Practice of Logic Programming (TPLP).
\end{abstract}

KEYWORDS: Logic programs, stable models, constraints

\section{Introduction}

In this paper, we introduce and study logic programs whose clauses are built of generalized atoms expressing constraints on sets. We propose a generalization of normal logic programming to this extended setting. Our generalization uses the assumption of the monotonicity of constraints and it employs the nondeterminism in deriving ways to satisfy constraints. In our approach the basic concepts, methods, semantics and results of normal logic programming generalize to the proposed context. Our work provides a theoretical framework to a recent extension of logic

* Parts of this paper appeared earlier in conference papers (Marek et al. 2004 Marek and Truszczyński 2004. 
programming with weight constraints (also known as pseudo-boolean constraints) (Niemelä et al. 1999, Simons et al. 2002), and to an earlier formalism of disjunctive logic programs with the semantics of possible models (Sakama and Inoue 1994), but applies to a much broader class of programs.

In the 1990s researchers demonstrated that normal logic programming with the stable-model semantics is an effective knowledge representation formalism. It provides solutions to problems arising in such contexts as planning, reasoning about actions, diagnosis and abduction, product configuration, and modeling and reasoning about preferences. Moreover, due to the emergence of fast methods to compute stable models (Niemelä and Simons 1997, Babovich and Lifschitz 2002, Lin and Zhao 2002, Leone et al. 2004), the importance of the formalism increased significantly as it became possible to use it not only as a modeling language but also as a practical computational tool. The contributions of (Baral 2003 Gelfond and Leone 2002) provide a detailed discussion of the formalism and its applications.

In the last few years, researchers proposed extensions of the language of normal logic programming with means to model constraints involving aggregate operations on sets. (Simons et al. 2002) proposed a formalism integrating logic programming with weight constraints, known in the SAT community as pseudo-boolean constraints, generalized the concept of stable models to this extended setting, and developed fast algorithms to compute them. (Denecker et al. 2001; Pelov et al. 2004), introduced a formalism allowing for more general aggregates. They extended to this new setting several semantics of normal logic programs, including the stable-model semantics and the well-founded semantics. A related recent work (Dell'Armi et al. 2003 Faber et al. 2004 Calimeri et al. 2005), incorporated aggregates into the formalism of disjunctive logic programs with the answer-set semantics. Yet another extension of normal logic programming has been proposed in (Marek and Remmel. 2004) as set-based constraints. Such extensions are important as they simplify the task of modeling problem specifications, typically result in more direct and concise encodings, and often significantly improve the computational effectiveness of the formalism as a problem-solving tool.

Our goal is to propose an abstract formalism of logic programs extended with means to model constraints on sets, preserving as much as possible analogies between our theory and normal logic programming. We introduce the notion of an abstract constraint and its linguistic counterpart - an abstract constraint atom. We then use abstract constraint atoms as building blocks of program clauses in the same way propositional atoms form clauses of normal logic programs. For the most part, we restrict our attention to monotone constraints, as monotonicity is essential for preserving the notion of a logic program as a computational device. We show that basic concepts, techniques, and results of normal logic programming have direct generalizations for the class of programs built of monotone abstract constraints. What distinguishes our work from other recent approaches to integrating logic programming with aggregates is that we allow constraint atoms in the heads of clauses, 
while formalisms proposed and studied in (Denecker et al. 2001 Pelov et al. 2004 Dell'Armi et al. 2003, Faber et al. 2004 Calimeri et al. 2005) do not ${ }^{1}$.

In many respects the theory we built in this paper mirrors closely an operatorbased treatment of normal logic programs. There is, however, a basic difference. Abstract constraint atoms are inherently nondeterministic. They can be viewed as shorthands for certain disjunctions and, in general, there are many ways to make an abstract constraint atom true. This nondeterminism has a consequence. The one-step provability operator, which generalizes the one-step provability operator of (van Emden and Kowalski 1976) defined for normal programs, is no longer deterministic. It assigns to an interpretation $M$ a set $T(M)$ of interpretations. Each interpretation in the set $T(M)$ is regarded as possible and equally likely outcome of applying the operator to $M$.

The nondeterministic one-step provability operator is a key tool in our work. It generalizes the one-step provability operator of (van Emden and Kowalski 1976). Thanks to close parallels between these two concepts, we are able to reconstruct operator-based characterizations of models, supported models, and the concept of a bottom-up computation for programs with abstract constraints that generalize Horn programs. We then extend to programs with abstract monotone constraints the definitions of the Gelfond-Lifschitz reduct and a stable model (Gelfond and Lifschitz 1988). We also distinguish and discuss the class of definite programs (programs with clauses whose heads can be satisfied in one way only). For these programs the one-step provability operator becomes deterministic and the theory of normal logic programming extends to deterministic programs without any significant change. In particular, it follows that normal logic programming with all its major 2-valued semantics can be viewed as a special case of logic programming with monotone abstract constraints.

In addition, we show that programs with abstract constraints provide a formal account of a class of logic programs with weight (pseudo-boolean) atoms introduced in (Simons et al. 2002). We call programs in the syntax proposed in that paper lparse programs. (Simons et al. 2002) defined for lparse programs the notion of a stable model and showed that lparse programs generalize normal logic programming with the stable-model semantics of Gelfond and Lifschitz (Gelfond and Lifschitz 1988). However, the notion of the reduct underlying the definition of a stable model given in (Simons et al. 2002) is different from that proposed in (Gelfond and Lifschitz 1988) and the precise nature of the relationship between normal logic programs and lparse programs was not clear.

Our work explicates this relationship. On one hand, the formalism of programs with abstract constraints parallels normal logic programming. In particular, major concepts, results and techniques in normal logic programming have counterparts in the setting of programs with abstract constraints. On the other hand, under some simple transformations, lparse programs can be viewed as a special case of programs with abstract constraints. Thus, through this connection, the theory of

\footnotetext{
${ }^{1}$ We note though that recently (Son et al. 2006) also considered programs with constraints in the heads of rules.
} 
normal logic programming can be lifted to the setting of lparse programs leading, in particular, to new characterizations of stable models of lparse programs.

Finally, we show that programs with monotone abstract constraints generalize the formalism of disjunctive logic programs with the semantics of possible models (Sakama and Inoue 1994). In fact, as we point out, several ideas that are stated in abstract terms in our paper have their roots in (Sakama and Inoue 1994).

\section{Basic concepts, motivation, examples}

We consider a language determined by a fixed set $A t$ of propositional atoms. An abstract constraint is a collection $C \subseteq \mathcal{P}(A t)$ (if $X$ is a set, by $\mathcal{P}(X)$ we denote the family of all subsets of $X)$. We say that elements of $C$ satisfy the constraint $C$ or have the property $C$. An abstract constraint atom (or ac-atom, for short) is an expression $C(X)$, where $X \subseteq A t$ is finite and $C$ is an abstract constraint. An ac-literal is an expression of the form $C(X)$ or $\operatorname{not}(C(X))$, where $C(X)$ is an ac-atom. We call $X$ the atom set of an ac-literal $A$ of the form $C(X) \operatorname{or} \operatorname{not}(C(X))$ and denote it by $\operatorname{aset}(A)$.

An intended meaning of an ac-atom $C(X)$ is to represent a requirement on subsets of $X$ that they must satisfy the constraint $C$. Formally, we interpret ac-atoms by means of propositional interpretations (truth assignments), which we represent as subsets of At: an atom $p$ is true in an interpretation $M \subseteq A t$ if $p \in M$, otherwise, $p$ is false in $M$. An interpretation $M \subseteq A t$ satisfies an ac-atom $C(X)$, written $M \models C(X)$, if $M \cap X \in C$ (that is, if the set of atoms in $X$ that are true in $M$ satisfies the constraint $C$ ). Otherwise, $M$ does not satisfy $C(X)$, written $M \not \models$ $C(X)$. In that case, we also say that $M$ satisfies the ac-literal $\operatorname{not}(C(X))$ and write $M \models \operatorname{not}(C(X))$. An ac-atom $C(X)$ is consistent if there is an interpretation $M$ such that $M \models C(X)$ or, equivalently, if $C$ contains at least one subset of $X$. We will now illustrate these concepts with several examples of common constraints.

Pseudo-boolean constraints. These constraints are also known as weight constraints. Given a real number $w$ and a function $W$, assigning to each atom in $A t$ a real number (its weight), a pseudo-boolean constraint $P B(w, W, \leq)$ imposes a restriction that "the total weight of atoms in a set is at least $w$ ". Formally, we set $P B(w, W, \leq)=\left\{A \subseteq A t: w \leq \sum_{a \in A} W(a)\right\}$ (comparison relations $<,>, \geq$ give rise to other types of weight constraints).

Cardinality constraints. They are pseudo-boolean constraints in which a bound $w$ is a non-negative integer and a weight of every atom is 1 . Throughout the paper, we use cardinality constraints to illustrate concepts we study. To simplify the notation and to make it consistent with the notation used in (Simons et al. 2002), we write $k X$ to denote an ac-atom $C(X)$, where $C=P B(k, W, \leq)$ and $W(a)=1$ for every $a \in A t$.

Product constraints. They differ from weight constraints in that they restrict the product of individual weights of atoms in allowed sets, depending on the type of the comparison relation used. Selecting the relation $\leq$ and assuming the same notation as before, we express product constraints as abstract constraints of the form $\Pi(w, W, \leq)=\left\{A \subseteq A t: w \leq \Pi_{a \in A} W(a)\right\}$. 
Maximum constraints. Given a weight function $W$ on the set of atoms and a real bound $w$, the maximum constraint restricts allowed sets of atoms to those with the maximum weight at least $w$. Formally, we express them as abstract constraints of the form $\operatorname{Max}(w, W, \leq)=\{A \subseteq A t: w \leq \max \{W(a): a \in A\}\}$ (or its variants, depending on the comparison relation).

Even- and odd-cardinality constraints. They impose a parity requirement on the cardinality of allowed sets. Formally, we express them as abstract constraints $E=\{A \subseteq A t:|A|$ is even $\}$ and $O=\{A \subseteq A t:|A|$ is odd $\}$.

Containment constraints. Such constraints require that allowed sets contain some prespecified configurations (subsets). We capture them by abstract constraints $C(\mathcal{A})$ that consist of all subsets of $A t$ that contain at least one set from a prespecified collection $\mathcal{A}$ of finite subsets of $A t$.

Each of these constraints determines associated ac-atoms. Let $A t=\left\{p_{1}, p_{2}, \ldots\right\}$ and let us consider a weight function $W$ such that for every integer $i \geq 1, W\left(p_{i}\right)=$ $i$. The expression $P B(6, W, \leq)$ is an example of a pseudo-boolean constraint. If we denote it by $C_{1}$, then $C_{1}\left(\left\{p_{1}, p_{2}, p_{5}, p_{6}\right\}\right)$ is an example of a pseudo-boolean constraint atom. A set $M \subseteq A t$ satisfies $C_{1}\left(\left\{p_{1}, p_{2}, p_{5}, p_{6}\right\}\right)$ if and only if the total weight of atoms in $M \cap\left\{p_{1}, p_{2}, p_{5}, p_{6}\right\}$ is at least 6 (that is, if and only if $M$ contains $p_{6}$, or $p_{5}$ together with at least one other atom). Similarly, $\operatorname{Max}(5, W, \leq)$ is an example of a maximum constraint and, if we denote it by $C_{2}, C_{2}\left(\left\{p_{2}, p_{4}, p_{6}, p_{8}\right\}\right)$ is a maximum constraint atom that enforces the restriction on sets of atoms to contain $p_{6}$ or $p_{8}$. An abstract constraint atom $E\left(\left\{p_{1}, p_{7}\right\}\right)$ ( $E$ stands for the evencardinality constraint) forces allowed sets of atoms to contain none or both of $p_{1}$ and $p_{7}$. All these constraint atoms are consistent. An atom $C_{3}\left(\left\{p_{1}, p_{2}, p_{3}\right\}\right)$, where $C_{3}=P B(7, w, \leq)$ is an example of an inconsistent constraint atom. No selection of atoms from $\left\{p_{1}, p_{2}, p_{3}\right\}$ satisfies it and, consequently, it has no models.

These examples demonstrate that abstract constraints and abstract constraint atoms express a broad range of common constraints. In this paper, we show that abstract constraint atoms can be combined into logic program clauses to represent even more complex constraints, and that much of the theory of normal logic programs generalizes to the extended setting.

\section{Logic programs built of $\mathcal{F}$-atoms}

Let $\mathcal{F}$ be a class of abstract constraints over $A t$. By an $\mathcal{F}$-atom we mean an abstract constraint atom $A(X)$ such that $A \in \mathcal{F}$ and $X \subseteq A t$. An $\mathcal{F}$-literal (or simply, a literal, if the context is clear) is an expression of the form $A(X)$ or $\operatorname{not}(A(X))$, where $A(X)$ is an $\mathcal{F}$-atom. An $\mathcal{F}$-clause is an expression

$$
A(X) \leftarrow B_{1}\left(X_{1}\right), \ldots, B_{m}\left(X_{m}\right), \operatorname{not}\left(C_{1}\left(Y_{1}\right)\right), \ldots, \operatorname{not}\left(C_{n}\left(Y_{n}\right)\right)
$$

where $A(X), B_{i}\left(X_{i}\right)$ and $C_{j}\left(Y_{j}\right)$ are $\mathcal{F}$-atoms. An $\mathcal{F}$-clause (10) is called a constraint clause if $A(X)$ is not consistent. An $\mathcal{F}$-program is a finite collection of $\mathcal{F}$-clauses ${ }^{2}$.

2 We note that the assumption of the finiteness of programs is not essential. The entire theory of $\mathcal{F}$-programs extends to the case when we admit infinite programs. However, additional means of 
If $r$ is a clause of the form (1), $A(X)$ is the head of $r$, denoted by $h d(r)$, and $X$ is the head set of $r$, denoted by $h \operatorname{set}(r)$. We also call the conjunction of literals $B_{1}\left(X_{1}\right), \ldots, B_{m}\left(X_{m}\right), \operatorname{not}\left(C_{1}\left(Y_{1}\right)\right), \ldots, \operatorname{not}\left(C_{n}\left(Y_{n}\right)\right)$, the body of $r$ and denote it by $b d(r)$. Occasionally, we use the same term to denote the set of all literals in the body of a clause. Finally, for an $\mathcal{F}$-program $P$, we $\operatorname{define} h \operatorname{set}(P)$ to be the union of sets $h \operatorname{set}(r)$, for $r \in P$.

An interpretation $M \subseteq$ At satisfies a set (conjunction) $L$ of literals, if it satisfies every literal in $L$. We say that $M$ satisfies an $\mathcal{F}$-clause $r$ if $M$ satisfies the head of the clause whenever it satisfies the body of $r$. Finally, $M$ satisfies an $\mathcal{F}$-program $P$ if it satisfies all clauses in $P$. We write $M \models L, M \models r$ and $M \models P$ to denote these three types of the satisfaction relation. We will often write "is a model of" instead of "satisfies". $\mathcal{F}$-programs that have models are called consistent.

Clauses of normal logic programs are typically regarded as computational devices: assuming that preconditions of a clause have been established, the clause provides a justification to establish (compute) its head. Crucial concepts behind formal accounts of that intuition are those of a Horn program, the corresponding bottom-up computation, and a least Herbrand model, which defines the result of the computation. Computations and their results are well defined due to the monotone behavior of Horn programs.

To extend normal logic programming to the class of programs with abstract constraint atoms, one needs a generalization of the class of Horn programs supporting an appropriate notion of a computation, with the results of computations playing the same role as that played by the least Herbrand model. In order to accomplish that, it is not enough simply to disallow the negation operator in the bodies of $\mathcal{F}$-clauses. It is also necessary to restrict the class of constraints to those that are monotone (that is, intuitively, once true in an interpretation, they remain true in every superset of it). Without that assumption, the monotonicity of normal Horn programs does not generalize and there is no straightforward way to define the concept of a computation. (We refer to (Marek 2005) for a study of properties of monotone constraints.)

Formally, we say that an abstract constraint $C$ is monotone if for every $A, A^{\prime} \subseteq$ At, if $A \in C$ and $A \subseteq A^{\prime}$ then $A^{\prime} \in C$ (in other words, monotone constraints are precisely upward-closed families of subsets of $A t)$. An abstract constraint atom $C(X)$ is monotone if its constraint $C$ is monotone.

\section{Proposition 1}

Let $C$ be an abstract monotone constraint over $A t, X \subseteq A t$, and let $M, M^{\prime} \subseteq A t$ be two interpretations. If $M \models C(X)$ and $M \subseteq M^{\prime}$, then $M^{\prime} \models C(X)$.

We note that if all the individual weights used by a weight function are nonnegative, the corresponding pseudo-boolean constraints are monotone. The maximum constraints are monotone for every weight function. On the other hand, we

proof, such as Zorn Lemma, may be required in some arguments (for instance, in the argument for the existence of minimal models of $\mathcal{F}$-programs). 
note that some common constraints, for instance, even- and odd-cardinality constraints $E$ and $O$, are not monotone.

From now on we restrict our attention to constraints that are monotone. We will write a monotone $\mathcal{F}$-clause and a monotone $\mathcal{F}$-program to make it explicit that all constraints in $\mathcal{F}$ are monotone.

An important consequence of the monotonicity assumption is that monotone $\mathcal{F}$-programs without constraint clauses have models (and so, also minimal models).

Proposition 2

Let $P$ be a monotone $\mathcal{F}$-program without constraint clauses. Then the set $A t$ of all atoms in the language is a model of $P$.

Proof

Let $r \in P$. Since the constraint $h d(r)$ is consistent, there is a set of atoms $M \subseteq A t$ such that $M \models h d(r)$. By the monotonicity of constraints in $\mathcal{F}, A t \models h d(r)$. Thus, $A t \models P$.

Another important consequence of the monotonicity assumption is that the concept of a Horn program has an obvious direct generalization.

\section{Definition 1}

A monotone $\mathcal{F}$-program that contains no occurrences of the operator not is a Horn $\mathcal{F}$-program.

Horn $\mathcal{F}$-programs defined in this way have many properties that generalize wellknown properties of normal Horn programs. We will state and prove several of them later in the paper.

\section{Nondeterministic one-step provability operator}

Following a fundamental idea underlying normal logic programming, we assign to $\mathcal{F}$-clauses a procedural interpretation, which views them as derivation clauses. In the discussion that follows we do not assume that constraints in $\mathcal{F}$ are monotone.

Intuitively, if an $\mathcal{F}$-clause $r$ has its body satisfied by some set of atoms $M$, then $r$ provides support for deriving from $M$ any set of atoms $M^{\prime}$ such that

1. $M^{\prime}$ consists of some atoms from the headset of $r$ ( $r$ provides no grounds for deriving atoms that do not appear in its headset)

2. $M^{\prime}$ satisfies the head of $r$ (since $r$ "fires", the constraint imposed by its head must hold).

Clearly, the process of deriving $M^{\prime}$ from $M$ by means of $r$ is nondeterministic in the sense that, in general, there are several sets that are supported by $r$ and $M$.

This interpretation of $\mathcal{F}$-clauses extends to $\mathcal{F}$-programs. Given an $\mathcal{F}$-program $P$ and a set of atoms $M$, each clause $r \in P$ such that $M$ satisfies the body of $r$ provides a support for a subset of the head set of $r$. The union, say $M^{\prime}$, of such sets - each supported by some clause $r$, with $r$ ranging over those clauses in $P$ whose body is satisfied by $M$ - can be viewed as "derived" from $M$ by means of 
$P$. In general, given $P$ and $M$, there may be several such derived sets. Thus, the notion of derivability associated with a program is nondeterministic, as in the case of individual clauses.

We describe formally this intuition of derivability in terms of a nondeterministic one-step provability operator. Before we give a precise definition, we note that by a nondeterministic operator on a set $D$ we mean any function $f: D \rightarrow \mathcal{P}(D)$. One can view the set $f(d)$ as the collection of all possible outcomes of applying $f$ to $d$ one of which, if $f(d) \neq \emptyset$, can be selected nondeterministically as the actual outcome of $f$. We emphasize that we allow $f(d)$ to be empty, that is, nondeterministic operators are, in general, partial - for some elements of the domain they do not assign any possible outcomes.

\section{Definition 2}

Let $\mathcal{F}$ be a class of constraints (not necessarily monotone). Let $P$ be an $\mathcal{F}$-program and let $M \subseteq A t$.

1. A clause $r \in P$ is $M$-applicable, if $M \models b d(r)$. We denote by $P(M)$ the set of all $M$-applicable clauses in $P$.

2. A set $M^{\prime}$ is nondeterministically one-step provable from $M$ by means of $P$, if $M^{\prime} \subseteq h \operatorname{set}(P(M))$ and $M^{\prime} \models h d(r)$, for every clause $r$ in $P(M)$.

3. The nondeterministic one-step provability operator $T_{P}^{\text {nd }}$, is a function from $\mathcal{P}(A t)$ to $\mathcal{P}(\mathcal{P}(A t))$ such that for every $M \subseteq A t, T_{P}^{n d}(M)$ consists of all sets $M^{\prime}$ that are nondeterministically one-step provable from $M$ by means of $P$.

Since an abstract constraint forming the head of an $\mathcal{F}$-clause may be inconsistent, there exist programs $P$ and interpretations $M \subseteq A t$ such that $T_{P}^{n d}(M)$ is empty.

The concepts introduced above have especially elegant properties for monotone $\mathcal{F}$-programs. First, to illustrate them, let us consider a simple example involving a program with cardinality constraints (cf. Section 2). The program discussed in this example is not a Horn program.

Example 1

Let $P$ be a program with cardinality constraints consisting of the following clauses:

$r_{1}=2\{a\} \leftarrow 2\{b, d\}$

$r_{2}=1\{b, c\} \leftarrow \operatorname{not}(1\{e\})$

$r_{3}=1\{a, d\} \leftarrow 2\{b, c\}$

We note that the cardinality atom in the head of the first clause is inconsistent.

Let us consider a set $M=\{b, c, e\}$. Since $M \not \forall 2\{b, d\}, r_{1}$ is not $M$-applicable. Similarly, $M \not \forall \operatorname{not}(1\{e\})$ and $r_{2}$ is not $M$-applicable, either. On the other hand, $M \models 2\{b, c\}$ and so, $r_{3}$ is $M$-applicable.

There are three subsets of $\{a, d\}$ that satisfy the constraint $1\{a, d\}$ in the head of the clause $r_{3}:\{a\},\{d\}$ and $\{a, d\}$. Thus, each of these sets is nondeterministically one-step provable from $M$ and, consequently,

$$
T_{P}^{n d}(M)=\{\{a\},\{d\},\{a, d\}\} .
$$

We also note that if $|M|=1$ and $e \notin M$ then $r_{2}$ is the only $M$-applicable clause in 
$P$. For such sets $M, T_{P}^{n d}(M)=\{\{b\},\{c\},\{b, c\}\}$. On the other hand, if $M$ contains both $b$ and $d$, then $r_{1}$ is $M$-applicable and since the head of $r_{1}$ is inconsistent, $T_{P}^{n d}(M)=\emptyset$ (no set is nondeterministically one-step provable from such a set $M$ ).

The example shows, in particular, that it may be the case that $T_{P}^{n d}(M)=\emptyset$. If, however, $P$ is a monotone $\mathcal{F}$-program without constraint clauses, then it is never the case.

Proposition 3

Let $P$ be a monotone $\mathcal{F}$-program without constraint clauses. For every $M \subseteq A t$, $h \operatorname{set}(P(M)) \in T_{P}^{n d}(M)$. In particular, $T_{P}^{n d}(M) \neq \emptyset$.

Proof

Let us consider $r \in P(M)$. Then, $h \operatorname{set}(P(M)) \cap h \operatorname{set}(r)=h \operatorname{set}(r)$. Since $h d(r)$ is consistent, it follows by the monotonicity of constraints in $\mathcal{F}$ that $h \operatorname{set}(r) \models h d(r)$. Thus, $h \operatorname{set}(P(M)) \models h d(r)$ and, consequently, $h \operatorname{set}(P(M)) \in T_{P}^{n d}(M)$.

The operator $T_{P}^{n d}$ plays a fundamental role in our research. It allows us to formalize the procedural interpretation of $\mathcal{F}$-clauses and identify several classes of models.

Our first result characterizes models of monotone $\mathcal{F}$-programs. Models of a normal logic program $P$ are prefixpoints of the one-step provability operator $T_{P}$ (van Emden and Kowalski 1976). This characterization lifts to the class of monotone $\mathcal{F}$-programs, with the operator $T_{P}^{n d}$ replacing $T_{P}$.

\section{Theorem 1}

Let $P$ be a monotone $\mathcal{F}$-program and let $M \subseteq A t$. The set $M$ is a model of $P$ if and only if there is $M^{\prime} \in T_{P}^{n d}(M)$ such that $M^{\prime} \subseteq M$.

\section{Proof}

Let $M$ be a model of $P$ and $M^{\prime}=M \cap h \operatorname{set}(P(M))$. Let $r \in P(M)$. Since $M$ is a model of $r, M \models h d(r)$. Clearly, $h \operatorname{set}(r) \subseteq h \operatorname{set}(P(M))$. Thus, $M \cap h \operatorname{set}(r)=$ $M^{\prime} \cap h \operatorname{set}(r)$ and, consequently, $M^{\prime} \models h d(r)$. It follows that $M^{\prime} \in T_{P}^{n d}(M)$. Since $M^{\prime} \subseteq M$, the assertion follows.

Conversely, let us assume that there is $M^{\prime} \in T_{P}^{n d}(M)$ such that $M^{\prime} \subseteq M$. Let $r \in P$ be a clause such that $M \models b d(r)$. Since $M^{\prime} \in T_{P}^{n d}(M), M^{\prime} \models h d(r)$. We recall that the constraint involved in $h d(r)$ is monotone (as we consider only monotone constraints). Thus, by Proposition 1 $M \models h d(r)$, as well. It follows that $M$ is a model of every clause in $P$ and, consequently, of $P$.

\section{Supported models of $\mathcal{F}$-programs}

For a set $M$ of atoms, we say that $M$-applicable clauses in an $\mathcal{F}$-program $P$ provide support to atoms in the heads of these clauses. In general, a model $M$ of an $\mathcal{F}$ program may contain elements that have no support in a program and $M$ itself, that is, cannot be derived from $M$ by means of clauses in the program. 
Example 2

Let $P$ be a program with cardinality constraints consisting of a single clause:

$$
1\{p, q\} \leftarrow \operatorname{not}(1\{q\}),
$$

where $p$ and $q$ are two different atoms. Let $M_{1}=\{q\}$. Clearly, $M_{1}$ is a model of $P$. However, $M_{1}$ has no support in $P$ and itself. Indeed, $T_{P}^{n d}\left(M_{1}\right)=\{\emptyset\}$ and so, $P$ and $M_{1}$ do not provide support for any atom. Similarly, another model of $P$, the set $M_{2}=\{p, s\}$, where $s \in A t$ is an atom different from $p$ and $q$, has no support in $P$ and itself. We have $T_{P}^{n d}\left(M_{2}\right)=\{\{p\},\{q\},\{p, q\}\}$ and so, $p$ has support in $P$ and $M_{2}$, but $s$ does not. Finally, the set $M_{3}=\{p\}$, which is also a model of $P$, has support in $P$ and itself. Indeed, $T_{P}^{n d}\left(M_{3}\right)=\{\{p\},\{q\},\{p, q\}\}$ and there is a way to derive $M_{3}$ from $P$ and $M_{3}$.

For $M$ to be a model of $P, M$ must satisfy the heads of all applicable clauses. To this end, $M$ needs to contain some of the atoms appearing in the headsets of these clauses (atoms with support in $M$ and $P$ ) and, possibly, also some atoms that do not have such support. Models that contain only atoms with support form an important class of models generalizing the class of supported models for normal logic programs (Clark 1978, Apt 1990).

\section{Definition 3}

Let $\mathcal{F}$ be a class of constraints (not necessarily monotone) and let $P$ be an $\mathcal{F}$ program. A set of atoms $M$ is a supported model of $P$ if $M$ is a model of $P$ and $M \subseteq h \operatorname{set}(P(M))$.

Supported models have the following characterization generalizing a characterization of supported models of normal logic programs as fixpoints of the van EmdenKowalski operator (the characterizing condition is commonly used as a definition of a fixpoint of a nondeterministic operator).

\section{Theorem 2}

Let $\mathcal{F}$ be a class of constraints (not necessarily monotone). Let $P$ be an $\mathcal{F}$-program. A set $M \subseteq A t$ is a supported model of $P$ if and only if $M \in T_{P}^{n d}(M)$.

\section{Proof}

If $M$ is a supported model of $P$ then it is a model of $P$ (by the definition). Moreover, $M \subseteq h \operatorname{set}(P(M))$. Thus, $M \in T_{P}^{n d}(M)$. Conversely, if $M \in T_{P}^{n d}(M)$, then $M \subseteq$ $h \operatorname{set}(P(M))$ and $M \models h d(r)$, for every $r \in P(M)$. Thus, $M \models r$, for every $r \in$ $P(M)$. If $r \in P \backslash P(M)$, then $M \forall b b d(r)$ and so, $M \models r$. Thus, $M$ is a model of $P$. Since $M \in T_{P}^{n d}(M)$ also implies $M \subseteq h \operatorname{set}(P(M)), M$ is a supported model of $P$.

In Section 8 we show that the use of the term supported for the class of models defined in this section is not a misnomer; supported models of $\mathcal{F}$-programs generalize supported models of normal logic programs. 


\section{Horn $\mathcal{F}$-programs}

For the concepts of the one-step provability and supported models we did not need a restriction to monotone constraints. To properly generalize the notion of a stable model, however, this restriction is essential. Thus, from this point on, we will consider only monotone $\mathcal{F}$-programs.

First, we will study Horn $\mathcal{F}$-programs (we recall that the notion of a Horn $\mathcal{F}$ program assumes that $\mathcal{F}$ consists of monotone constraints only) viewing them as representations of certain nondeterministic computational processes. We will later use the results of this section to extend to the class of $\mathcal{F}$-programs the concept of a stable model.

\section{Definition 4}

Let $P$ be a Horn $\mathcal{F}$-program. A $P$-computation is a sequence $\left(X_{n}\right)_{n=0,1, \ldots}$ such that $X_{0}=\emptyset$ and, for every non-negative integer $n$ :

1. $X_{n} \subseteq X_{n+1}$, and

2. $X_{n+1} \in T_{P}^{n d}\left(X_{n}\right)$.

Given a computation $t=\left(X_{n}\right)_{n=0,1, \ldots}$, we call $\bigcup_{n=0}^{\infty} X_{n}$ the result of the computation $t$ and denote it by $R_{t}$.

Our stipulations that $P$-computations have length $\omega$ does not restrict the generality. Since atom sets of ac-atoms are finite, if a clause is applicable with respect to the result of the computation, it is applicable at some step $n$ of the computation. Consequently, like in the case of normal Horn programs, all possible results of computations of arbitrary transfinite lengths can be reached in $\omega$ steps, even in the case of infinite programs.

Results of computations are supported models.

Theorem 3

Let $P$ be a Horn $\mathcal{F}$-program and let $t$ be a $P$-computation. Then $R_{t}$ is a supported model of $P$, that is, $R_{t}$ is a model of $P$ and $R_{t} \subseteq \operatorname{hset}\left(P\left(R_{t}\right)\right)$.

Proof

Let $t=\left(X_{n}\right)_{n=0,1, \ldots}$. Clearly $X_{0}=\emptyset \subseteq h \operatorname{set}\left(P\left(R_{t}\right)\right)$. Let $n$ be a non-negative integer. Since $X_{n+1} \in T_{P}^{n d}\left(X_{n}\right), X_{n+1} \subseteq \operatorname{hset}\left(P\left(X_{n}\right)\right)$. Since $P$ is a Horn $\mathcal{F}$-program, it follows that if $r \in P, X \subseteq Y$ and $X \models b d(r)$, then $Y \models b d(r)$. Thus, since $X_{n} \subseteq R_{t}$, we have

$$
X_{n+1} \subseteq h \operatorname{set}\left(P\left(X_{n}\right)\right) \subseteq h \operatorname{set}\left(P\left(R_{t}\right)\right)
$$

By induction, $R_{t}=\bigcup_{n=0}^{\infty} X_{n} \subseteq \operatorname{hset}\left(P\left(R_{t}\right)\right)$.

Conversely, let us consider a clause $r \in P$. If $R_{t} \not \models b d(r)$ then $R_{t} \models r$. Let us then assume that $R_{t} \models b d(r)$. Since $r$ has finitely many $\mathcal{F}$-atoms in the body, and since each $\mathcal{F}$-atom is of the form $C(X)$, where $X$ is finite, there is a non-negative integer $i$ such that $X_{i} \models b d(r)$. By the definition of a $P$-computation, $X_{i+1} \in T_{P}^{n d}\left(X_{i}\right)$. Thus, $X_{i+1} \models h d(r)$ and, since $X_{i+1} \subseteq R_{t}, R_{t} \models h d(r)$ (by the monotonicity of $h d(r))$. It follows that $R_{t} \models r$ in the case when $R_{t} \models b d(r)$, as well. Thus, $R_{t}$ is 
a model of $P$. Since $R_{t}$ is a model of $P$ and $R_{t} \subseteq h \operatorname{set}\left(P\left(R_{t}\right)\right), R_{t}$ is a supported model of $P$.

We will now show that having a model (being consistent) is a necessary and sufficient condition for a Horn $\mathcal{F}$-program to have a computation. To this end, we will first introduce a certain class of computations.

\section{Definition 5}

Let $M$ be a model of $P$. A canonical $P$-computation with respect to $M$ is a sequence $t^{P, M}=\left(X_{n}^{P, M}\right)_{n=0,1, \ldots}$ defined as follows:

1. $X_{0}^{P, M}=\emptyset$ and,

2. $X_{n+1}^{P, M}=h \operatorname{set}\left(P\left(X_{n}^{P, M}\right)\right) \cap M$, for every $n \geq 0$.

We observe that canonical computations involve no nondeterminism. At each stage there is exactly one way in which we can continue. This continuation is determined by the model $M$. Before we proceed further, we illustrate the concept of a canonical computation with a simple example.

\section{Example 3}

Let us assume that $A t=\{a, b, c, d\}$ and let us consider a Horn program with cardinality constraints, say $P$, consisting of the following clauses:

$r_{1}=1\{a, d\} \leftarrow 2\{b, d\}$

$r_{2}=1\{b, c\} \leftarrow$

$r_{3}=1\{a\} \leftarrow 2\{b, c\}$

Let $M=\{a, b, c, d\}$. It is easy to check that $M$ is a model of $P$ (it also follows from Proposition 2 as the constraint atoms in the heads of clauses in $P$ are consistent).

We will now construct a canonical $P$-computation with respect to $M$. By the definition $X_{0}^{P, M}=\emptyset$. The only $X_{0}^{P, M}$-applicable clause in $P$ is $r_{2}$. Since $\{b, c\} \cap M=$ $\{b, c\}, X_{1}^{P, M}=\{b, c\}$. The clauses $r_{2}$ and $r_{3}$ are $X_{1}^{P, M}$-applicable and $r_{1}$ is not. Since $\{a\} \cap M=\{a\}$ and $\{b, c\} \cap M=\{b, c\}, X_{2}^{P, M}=\{a, b, c\}$. Since $r_{2}$ and $r_{3}$ are the only $X_{2}^{P, M}$-applicable clauses in $P$, it follows that $X_{k}^{P, M}=X_{2}^{P, M}$, for $k=3,4, \ldots$.

By the definition, the union of all sets in the canonical computation is included in $M$. Our example demonstrates that canonical computations with respect to $M$, in general, do not reconstruct all of $M$.

The use of the term $P$-computation in Definition 5 is justified. The following theorem shows that the sequence $t^{P, M}$ is indeed a $P$-computation.

\section{Theorem 4}

Let $P$ be a Horn $\mathcal{F}$-program and let $M \subseteq A t$ be a model of $P$. Then the sequence $t^{P, M}$ is a $P$-computation. 
Proof

We need to show that the conditions (1) and (2) from the definition of a $P$ computation hold for the sequence $t^{P, M}$. To prove (1), we proceed by induction on $n$. For $n=0$, the condition (1) is, clearly, satisfied. Let us assume that for some non-negative integer $n, X_{n}^{P, M} \subseteq X_{n+1}^{P, M}$ holds. Then

$$
h \operatorname{set}\left(P\left(X_{n}^{P, M}\right)\right) \subseteq h \operatorname{set}\left(P\left(X_{n+1}^{P, M}\right)\right) .
$$

It follows that

$$
X_{n+1}^{P, M}=h \operatorname{set}\left(P\left(X_{n}^{P, M}\right)\right) \cap M \subseteq h \operatorname{set}\left(P\left(X_{n+1}^{P, M}\right)\right) \cap M=X_{n+2}^{P, M} .
$$

To prove (2), let us consider a non-negative integer $n$. By the definition, $X_{n+1}^{P, M} \subseteq$ $h \operatorname{set}\left(P\left(X_{n}^{P, M}\right)\right)$. It remains to prove that $X_{n+1}^{P, M} \models P\left(X_{n}^{P, M}\right)$. Let $r \in P\left(X_{n}^{P, M}\right)$. Then $X_{n}^{P, M} \models b d(r)$ and, since $X_{n}^{P, M} \subseteq M, M \models b d(r)$. We recall that $M$ is a model of $P$. Thus, $M \models h d(r)$. It follows that $M \cap h \operatorname{set}(r) \models h d(r)$ and, consequently, $M \cap h \operatorname{set}\left(P\left(X_{n}^{P, M}\right)\right) \models h d(r)$. Since $X_{n+1}^{P, M}=M \cap h \operatorname{set}\left(P\left(X_{n}^{P, M}\right)\right)$, it follows that $X_{n+1}^{P, M} \models P\left(X_{n}^{P, M}\right)$.

We now have the following corollary to Theorems 3 and 4 that characterizes Horn $\mathcal{F}$-programs that have computations.

Corollary 1

Let $P$ be a Horn $\mathcal{F}$-program. Then, $P$ has a model if and only if it has a $P$ computation. In particular, every Horn $\mathcal{F}$-program $P$ without constraint clauses possesses at least one $P$-computation.

Proof

If $M$ is a model of $P$ then the canonical computation $t^{P, M}$ is a $P$-computation (Theorem (4). Conversely, if $P$ has a $P$-computation $t$, then $R_{t}$ is a model of $P$ (Theorem 31). The second part of the assertion follows from the fact that Horn $\mathcal{F}$-programs without constraint clauses have models (Proposition 2).

We use the concept of a computation to identify a certain class of models of Horn $\mathcal{F}$-programs.

\section{Definition 6}

Let $P$ be a Horn $\mathcal{F}$-program. We say that a set of atoms $M$ is a derivable model of $P$ if there exists a $P$-computation $t$ such that $M=R_{t}$.

Derivable models play in our theory a role analogous to that of the least model of a normal Horn program. The basic analogy is that they are the results of bottom-up computations, as is the case for the least model of a normal Horn program.

Theorems 3 and 4 entail several properties of Horn $\mathcal{F}$-programs, their computations and models. We gather them in the following corollary. Properties (1) and (3)

- (6) generalize properties of the least model of a normal Horn logic program. 
Corollary 2

Let $P$ be a Horn $\mathcal{F}$-program. Then:

1. If $P$ is consistent then $P$ has at least one derivable model.

2. For every model $M$ of $P$ there is a largest derivable model $M^{\prime}$ of $P$ such that $M^{\prime} \subseteq M$.

3. A model $M$ of $P$ is derivable if and only if $M=R_{t^{P, M}}$.

4. If $P$ contains no constraint clauses then $P$ has a largest derivable model.

5. Every minimal model of $P$ is derivable.

6. Every derivable model of $P$ is a supported model of $P$.

Proof

(1) Since $P$ has a model, it has a $P$-computation (Theorem 4). The result of this computation is a model of $P$ (Theorem B). By the definition, this model is derivable.

(2) Let $M$ be a model of $P$ and let $t=\left(X_{n}\right)_{n=0,1, \ldots}$ be the canonical $P$-computation for $M$. Then, $R_{t}$ is a derivable model of $P$ and $R_{t} \subseteq M$. Let $s=\left(Y_{n}\right)_{n=0,1, \ldots}$ be a $P$-computation such that $R_{s} \subseteq M$. Clearly, we have $Y_{0} \subseteq X_{0}$. Let us consider an integer $n>0$ and let us assume that the inclusion $Y_{n-1} \subseteq X_{n-1}$ holds. Since $R_{s} \subseteq M, Y_{n} \subseteq M$. Thus, by the definition of a $P$-computation,

$$
Y_{n} \subseteq h \operatorname{set}\left(P\left(Y_{n-1}\right)\right) \cap M \text {. }
$$

Since $P$ is a Horn $\mathcal{F}$-program and since we have $Y_{n-1} \subseteq X_{n-1}, h \operatorname{set}\left(P\left(Y_{n-1}\right)\right) \subseteq$ $h \operatorname{set}\left(P\left(X_{n-1}\right)\right)$. Thus,

$$
Y_{n} \subseteq h \operatorname{set}\left(P\left(X_{n-1}\right)\right) \cap M=X_{n} .
$$

It follows now by induction that $R_{s} \subseteq R_{t}$. Thus, $R_{t}$ is the largest derivable model contained in $M$.

(3) Let $M$ be a model of $P$. The argument we used in (2) shows that the result of the canonical computation from $P$ with respect to $M$ is the greatest derivable model contained in $M$. If $M$ is derivable, then $M=R_{t^{P, M}}$. The converse implication follows by the definition.

(4) The set $A t$ is a model of $P$. Let $R$ be the result of the canonical $P$-computation for At. Clearly, $R$ is a derivable model of $P$. We will show that every derivable model of $P$ is a subset of $R$. Let $M$ be a derivable model of $P$. By (3), $M$ is the result of a canonical computation for $M$. Since $M \subseteq A t$, it follows by an induction argument that for every non-negative integer $n, X_{n}^{P, M} \subseteq X_{n}^{P, A t}$ (we omit the details, as the argument is similar to that in the proof of (2)). Consequently, $M \subseteq R$.

(5) This assertion follows directly from (2).

(6) This assertion follows directly from Theorem 3

Despite analogies with the least model of a normal Horn program, derivable models are not, in general, minimal. For instance, a program with cardinality constraints consisting of a single clause

$$
1\{a, b\} \leftarrow
$$

has three derivable models: $\{a\},\{b\}$ and $\{a, b\}$, only two of which are minimal. 
Horn $\mathcal{F}$-programs generalize Horn normal logic programs (see Section 8 for details.). For a Horn $\mathcal{F}$-programs without constraint clauses, the canonical computation with respect to the set of all atoms is a counterpart to the bottom-up computation determined by a normal Horn program.

\section{Stable models of monotone $\mathcal{F}$-programs}

We will now use the results of the two previous sections to introduce and study the class of stable models of monotone $\mathcal{F}$-programs.

\section{Definition 7}

Let $P$ be a monotone $\mathcal{F}$-program and let $M \subseteq A t$. The reduct of $P$ with respect to $M, P^{M}$ in symbols, is a Horn $\mathcal{F}$-program obtained from $P$ by (1) removing from $P$ every $\mathcal{F}$-clause containing in the body a literal not $(A)$ such that $M \models A$, and (2) removing all literals of the form $\operatorname{not}(A)$ from all the remaining clauses in $P$. A set of atoms $M$ is a stable model of $P$ if $M$ is a derivable model of the reduct $P^{M}$.

The following result is easy to show (and so we omit its proof) but useful.

\section{Lemma 1}

Let $P$ be a monotone $\mathcal{F}$-program. If $M$ is a model of $P$, then $M$ is a model of $P^{M}$.

\section{Example 4}

We illustrate the concept of stable models of monotone $\mathcal{F}$-programs with examples underlining some aspects of their properties. The class $\mathcal{F}$ we use in this example consists of all cardinality constraints which, we recall, are monotone (Section 2).

Let $P$ be a program consisting of the following two clauses:

$1\{a, b\} \leftarrow 1\{d\}, \operatorname{not}(1\{b, c\})$

$1\{a, d\} \leftarrow$

We will now investigate properties of some sets with respect to this program.

(1) The set $M_{1}=\emptyset$ is not a model of our program $P$. As we will see soon (Proposition 4), stable models are supported models and, consequently, also models. Thus $\emptyset$ is not a stable model of $P$.

(2) The set $M_{2}=\{a, b, c\}$ is a model of $P$. But $M_{2}$ is not a stable model of $P$. Indeed, let us compute $P^{M_{2}}$. It consists of just one clause: $1\{a, d\} \leftarrow$. Since $M_{2}$ is not a derivable model of $P^{M_{2}}$ (it contains an atom not occurring in any head of the clause of $\left.P^{M_{2}}\right), M_{2}$ is not a stable model of $P$

(3) The set $M_{3}=\{a, d\}$ is a stable model of $P$. The reduct $P^{M_{3}}$ consists of two clauses:

$1\{a, b\} \leftarrow 1\{d\}$

$1\{a, d\} \leftarrow$

The sequence $\emptyset,\{a, d\},\{a, d\}, \ldots$ is a $P^{M_{3}}$-computation. Thus, $M_{3}$ is a derivable model of $P^{M_{3}}$ and hence $M_{3}$ is a stable model of $P$

(4) The set $M_{4}=\{a\}$ is a stable model of $P$. The reduct $P^{M_{4}}$ consists of two clauses: 
$1\{a, b\} \leftarrow 1\{d\}$

$1\{a, d\} \leftarrow$

The sequence $\emptyset,\{a\},\{a\}, \ldots$ is a $P^{M_{4}}$-computation. Thus $\{a\}$ is a stable model of $P$.

In our example $M_{4} \subset M_{3}$. Thus, in contrast to normal logic programs (but not to lparse programs), stable models of abstract constraint programs can nest. That is, they do not satisfy the antichain (minimality with respect to inclusion) property.

The program $P$ that we considered above has stable models. It is easy to construct examples of programs that have no stable models. For instance, a program consisting of just one clause: $2\{a, b, c\} \leftarrow \operatorname{not}(1\{a, b\})$ has models but no stable models.

Stable models of a monotone $\mathcal{F}$-program $P$ are indeed models of $P$. Thus, the use of the term "model" in their name is justified. In fact, a stronger property holds: stable models of monotone $\mathcal{F}$-programs are supported. This again generalizes a well-known property of normal logic programs ${ }^{3}$.

\section{Proposition 4}

Let $P$ be a monotone $\mathcal{F}$-program. If $M \subseteq A t$ is a stable model of $P$ then $M$ is a supported model of $P$.

\section{Proof}

First, let us observe that it follows directly from the corresponding definitions that $T_{P}^{n d}(M)=T_{P M}^{n d}(M)$. Next, since the set $M$ is a derivable model of $P^{M}, M$ is a supported model of $P^{M}$ (Corollary 2(6)). Thus, by Theorem 2] $M \in T_{P^{M}}^{n d}(M)$ and, consequently, $M \in T_{P}^{n d}(M)$. It follows that $M$ is a supported model of $P$.

With the notion of a stable model in hand, we can strengthen Theorem 3

\section{Theorem 5}

Let $P$ be a Horn $\mathcal{F}$-program. A set of atoms $M \subseteq A t$ is a derivable model of $P$ if and only if $M$ is a stable model of $P$.

Proof

The assertion is a direct consequence of the fact that for every Horn $\mathcal{F}$-program $P$ and for every set of atoms $M, P=P^{M}$.

We will now prove yet another result that generalizes a property of stable models of normal logic programs (cf. work on extending the semantics of stable models to logic programs with integrity constraints (Lifschitz 1996)).

\footnotetext{
${ }^{3}$ Incidentally, in the case of programs with weight constraints in the lparse syntax, no such property has been established as supported models have not been defined for that formalism.
} 
Proposition 5

Let $P$ and $Q$ be two monotone $\mathcal{F}$-programs.

1. If $M$ is a stable model of $P$ and a model of $Q$ then $M$ is a stable model of $P \cup Q$.

2. If $Q$ consists of constraint clauses and $M$ is a stable model of $P \cup Q$ then $M$ is a stable model of $P$.

Proof

(1) Since $M$ is a stable model of $P, M$ is a derivable model of $P^{M}$. By Corollary 22 3), $M$ is the result of the canonical $P^{M}$-computation with respect to $M$. Since $M$ is a model of $P \cup Q$, by Lemma $1 M$ is a model of $(P \cup Q)^{M}=P^{M} \cup Q^{M}$. Therefore, the canonical $\left(P^{M} \cup Q^{M}\right)$-computation with respect to $M$ is well defined. Its result is clearly contained in $M$. On the other hand, it contains the result of the canonical $P^{M}$-computation with respect to $M$, which is $M$. Therefore, the result of the canonical $\left(P^{M} \cup Q^{M}\right)$-computation with respect to $M$ is $M$. Thus, $M$ is a derivable model of $(P \cup Q)^{M}$ and a stable model of $P \cup Q$.

(2) Since $M$ is a stable model of $P \cup Q, M$ is the result of a $(P \cup Q)^{M}$-computation, say $t$. Since $Q$ consists of constraint clauses, $t$ is a $P^{M}$-computation (constraint clauses, having inconsistent heads, do not participate in computations). Thus, $M$ is also a result of a $P^{M}$-computation, that is, $M$ is a stable model of $P$.

\section{Monotone $\mathcal{F}$-programs and normal logic programming}

The main goal of this paper is to propose a way to integrate abstract constraints into normal logic programming. In this section, we show that our formalism of $\mathcal{F}$ programs contains normal logic programming (modulo a very simple encoding) so that all major two-valued semantics are preserved.

To this end, let us consider an abstract constraint:

$$
P B=\{X \subseteq A t: X \neq \emptyset\} .
$$

We note that $P B$ is identical with the pseudo-boolean constraint (we introduced pseudo-boolean constraints in Section 2):

$$
P B=P B(1, W, \leq),
$$

where $W$ is a weight function on $A t$ such that $W(a)=1$, for every $a \in A t$. Clearly, the constraint $P B$ is monotone. We will show that normal logic programs can be encoded as $\{P B\}$-programs or, more generally, as monotone $\mathcal{F}$-programs, for every class $\mathcal{F}$ of monotone abstract constraints such that $P B \in \mathcal{F}$. In what follows, if $a \in A t$, we will write $P B(a)$ for a $\{P B\}$-atom $P B(\{a\})$.

We note that for every $a \in A t$ and every interpretation $M \subseteq A t, M \models a$ if and only if $M \models P B(a)$. That is, a propositional atom $a$ is logically equivalent to an abstract constraint atom $P B(a)$. This equivalence suggests an encoding of a normal logic program $P$ as $\{P B\}$-program $P^{p b}$. Namely, if $\mathrm{r}$ is a normal logic program clause

$$
a \leftarrow b_{1}, \ldots, b_{m}, \operatorname{not}\left(c_{1}\right), \ldots, \operatorname{not}\left(c_{n}\right)
$$


we define $r^{p b}$ to be a $\{P B\}$-clause

$$
P B(a) \leftarrow P B\left(b_{1}\right), \ldots, P B\left(b_{m}\right), \operatorname{not}\left(P B\left(c_{1}\right)\right), \ldots, \operatorname{not}\left(P B\left(c_{n}\right)\right) .
$$

For a normal logic program $P$, we define $P^{p b}=\left\{r^{p b}: r \in P\right\}$. By our earlier comments, $P^{p b}$ is a monotone $\mathcal{F}$-program, for every class of monotone constraint atoms containing the constraint $P B$.

We note that due to the equivalence of $a$ and $P B(a)$, which we discussed above, for every interpretation $M \subseteq A t$ we have

$$
M \models b d(r) \text { if and only if } M \models b d\left(r^{p b}\right) .
$$

(here and in other places we use symbols such as $b d(r), h d(r)$ and $h d(P)$ also in the context of normal logic programs, and assume their standard meaning).

Our first result involves operators associated with programs. Let $P$ be a normal logic program. We recall that the one-step provability operator $T_{P}$ (van Emden and Kowalski 1976) is defined as follows: for every $M \subseteq A t$,

$$
T_{P}(M)=\{h d(r): r \in P \text { and } M \models b d(r)\} .
$$

We have the following basic property of the translation $P \mapsto P^{p b}$.

Proposition 6

Let $P$ be a normal logic program. Then for every $M \subseteq A t(P), T_{P^{p b}}^{n d}(M)=\left\{T_{P}(M)\right\}$.

Proof

We will write $r$ and $r^{\prime}$ for a pair of corresponding clauses in $P$ and $P^{p b}$. That is, if $r \in P$ then $r^{\prime}=r^{p b}$ is the counterpart of $r$ in $P^{p b}$. Conversely, if $r^{\prime} \in P^{p b}, r$ is the clause in $P$ such that $r^{p b}=r^{\prime}$. Clearly, we have $h \operatorname{set}\left(r^{\prime}\right)=\{h d(r)\}$.

By the equivalence (2), a clause $r \in P$ is $M$-applicable if and only if $r^{\prime}$ is $M$ applicable. Thus, we have

$$
h \operatorname{set}\left(P^{p b}(M)\right)=h d(P(M))=T_{P}(M) .
$$

Let $r^{\prime} \in P^{p b}(M)$ and let $a=h d(r)$. It follows that $r \in P(M)$ and $a \in T_{P}(M)$. Since $h d\left(r^{\prime}\right)=P B(a), T_{P}(M) \models h d\left(r^{\prime}\right)$. Thus, $T_{P}(M)$ is one-step nondeterministically provable from $M$ and $P^{p b}$, that is, $T_{P}(M) \in T_{P^{p b}}^{n d}(M)$.

Next, let us consider $M^{\prime} \in T_{P p b}^{n d}(M)$. By the definition, $M^{\prime} \subseteq h \operatorname{set}\left(P^{p b}(M)\right)$. Thus, by (3), we have $M^{\prime} \subseteq T_{P}(M)$. Let us now consider $a \in T_{P}(M)$. It follows that there is a clause $r \in P(M)$ such that $h d(r)=a$. Consequently, $r^{\prime} \in P^{p b}(M)$ and $h d\left(r^{\prime}\right)=P B(a)$. Since $M^{\prime} \in T_{P^{p b}}^{n d}(M), M^{\prime} \models h d\left(r^{\prime}\right)$. Thus, $a \in M^{\prime}$. It follows that $M^{\prime}=T_{P}(M)$ and, consequently, $T_{P p^{b}}^{n d}(M)=\left\{T_{P}(M)\right\}$.

This result entails a proposition concerning Horn programs.

\section{Proposition 7}

Let $P$ be a normal Horn logic program. Then $M$ is a least model of $P$ if and only if $M$ is a derivable model of $P^{p b}$. 
Proof

We first observe that the sequence $\left\{T_{P} \uparrow n(\emptyset)\right\}_{n=0,1, \ldots}$ is a $P^{p b}$-computation (one can show this by an easy inductive argument, using the relationship between $T_{P}$ and $T_{P^{p b}}^{n d}$ established by Proposition [6] Since $M$ is the limit of the sequence $\left\{T_{P} \uparrow\right.$ $n(\emptyset)\}_{n=0,1, \ldots}, M$ is a derivable model of $P^{p b}$.

Conversely, if $M$ is a derivable model of $P^{p b}$, then $M$ is the result of a derivation $\left\{X_{n}\right\}_{n=0,1, \ldots}$ from $P^{p b}$. Thus, for every $n=0,1, \ldots, X_{n+1} \in T_{P p b}^{n d}\left(X_{n}\right)$. By Proposition 6 $X_{n+1}=T_{P}\left(X_{n}\right)$. Since $X_{0}=\emptyset$, it follows that for every $n=0,1, \ldots$, $X_{n}=T_{P} \uparrow n(\emptyset)$. Consequently, $M=\bigcup_{n=0}^{\infty} T_{P} \uparrow n(\emptyset)$ and so, $M$ is the least model of $P$.

We can now prove the main result of this section demonstrating that the embedding $P \mapsto P^{p b}$ preserves all the semantics considered in the paper.

\section{Theorem 6}

Let $P$ be a normal logic program and let $M$ be a set of atoms. Then $M$ is a model (supported model, stable model) of $P$ if and only if $M$ is a model (supported model, stable model) of $P^{p b}$.

\section{Proof}

It is well known that $M$ is a model of $P$ if and only if $T_{P}(M) \subseteq M$ (Apt 1990). By Proposition [6] the latter condition is equivalent to the condition that there is $M^{\prime} \in T_{P p b}^{n d}(M)$ such that $M^{\prime} \subseteq M$. By Theorem 1his last condition is equivalent to $M$ being a model of $P^{p b}$. Thus, $M$ is a model of $P$ if and only if $M$ is a model of $P^{p b}$.

The proof for the case of supported models is essentially the same. It relies on the fact that $M$ is a supported model of $P$ if and only if $M=T_{P}(M)$ (Apt 1990) and uses Proposition [6] and Theorem 2

Let us assume now that $M$ is a stable model of $P$. It follows that $M$ is the least model of $P^{M}$. By Proposition [7 $M$ is a derivable model of $\left[P^{M}\right]^{p b}$. It follows from the definitions of the reducts of normal logic programs and $\{P B\}$-programs that $\left[P^{M}\right]^{p b}=\left[P^{p b}\right]^{M}$. Thus, $M$ is a stable model of $P^{p b}$. The converse implication can be proved in the same way.

There are other ways to establish a connection between normal logic programs and programs with abstract constraints. We will now define a class of monotone $\mathcal{F}$-programs, which offers a most direct extension of normal logic programming.

Definition 8

An $\mathcal{F}$-atom $C(X)$ is definite if $X$ is a minimal element in $C$. An $\mathcal{F}$-clause $r$ is definite if $h d(r)$ is a definite $\mathcal{F}$-atom. An $\mathcal{F}$-program is definite if every clause in $P$ is definite.

We use the term definite following the logic programming tradition (cf. (van Emden and Kowalski 1976), for instance), where it is used for clauses whose heads provide "definite" information (as opposed to being disjunctions and so listing several possible alternatives). 
Example 5

Let $\mathcal{F}$ consist of two monotone constraints, $C_{1}$ and $C_{2}$ where:

$$
C_{1}=\{X \subseteq A t:\{a, b\} \subseteq X \text { or }\{a, c\} \subseteq X \text { or }|X| \text { is infinite }\}
$$

and

$$
C_{2}=\{X \subseteq A t:\{d, e\} \subseteq X\}
$$

The constraint $C_{1}$ has two minimal elements: $\{a, b\}$ and $\{a, c\}$. The constraint $C_{2}$ has just one minimal element: $\{d, e\}$.

These two monotone constraints generate the following three definite atoms: $C_{1}(\{a, b\}), C_{1}(\{a, c\})$, and $C_{2}(\{d, e\})$. An $\mathcal{F}$-program consisting of the following clauses is definite:

$C_{1}(\{a, b\}) \leftarrow$

$C_{1}(\{a, c\}) \leftarrow C_{1}(\{a, b, c\}), \operatorname{not}\left(C_{2}(\{a, b, d, e\})\right)$

$C_{2}(\{d, e\}) \leftarrow$

We note that some monotone constraints do not yield any definite constraint atoms. It happens when they have no finite minimal elements. A constraint $C$ consisting of all infinite subsets of $A t$ offers a specific example.

Definite $\mathcal{F}$-atoms have the following simple properties.

\section{Proposition 8}

Let $X \subseteq A t$ and let $C(X)$ be a definite $\mathcal{F}$-atom. Then $C(X)$ is consistent and, for every $M \subseteq A t, M \models C(X)$ if and only if $X \subseteq M$.

Proof

If $M \models C(X)$ then $M \cap X \in C$. Since $C(X)$ is a definite $\mathcal{F}$-atom, $X$ is a minimal element in $C$. It follows that $M \cap X=X$ and so, $X \subseteq M$. Conversely, if $X \subseteq M$ then $M \cap X=X$. Since $X \in C, M \cap X \in C$. Thus, $M \models C(X)$. This argument proves the second part of the assertion. In particular, it follows that $X \models C(X)$. Thus, $C(X)$ is consistent.

The intuition behind the notion of a definite $\mathcal{F}$-atom is now clear. Given a definite $\mathcal{F}$-program and an interpretation $M$, there is always a way to satisfy the heads of all $M$-applicable clauses (due to consistency of definite $\mathcal{F}$-atoms). Moreover, there is only one way to do so if we want only to use atoms appearing in the headsets of $M$-applicable clauses (due the the second property from Proposition 8 ). Thus, computing with definite $\mathcal{F}$-programs does not involve nondeterminism. Indeed, we have the following result.

Proposition 9

Let $P$ be a definite $\mathcal{F}$-program. Then, for every set of atoms $M,\left|T_{P}^{n d}(M)\right|=1$. 
Proof

Let $r \in P(M)$. Since $h d(r)$ is a definite $\mathcal{F}$-atom, then $h \operatorname{set}(r) \models h d(r)$. We now observe that $h \operatorname{set}(r) \subseteq h \operatorname{set}(P(M))$. Thus, for every $r \in P(M), \operatorname{hset}(P(M)) \models$ $h d(r)$. By the definition of the one-step nondeterministic provability, $h \operatorname{set}(P(M)) \in$ $T_{P}^{n d}(M)$. Thus, $\left|T_{P}^{n d}(M)\right| \geq 1$.

Next, let us consider $M^{\prime} \in T_{P}^{n d}(M)$. From the definition of $T_{P}^{n d}(M)$, it follows that $M^{\prime} \subseteq h \operatorname{set}(P(M))$. To prove the converse inclusion, let $r \in P(M)$. Again by the definition of $T_{P}^{n d}(M)$, we have that $M^{\prime} \models h d(r)$. Since $h d(r)$ is a definite $\mathcal{F}$-atom, Proposition 8 implies that $h \operatorname{set}(r) \subseteq M^{\prime}$. Thus, $h \operatorname{set}(P(M)) \subseteq M^{\prime}$.

It follows that $h \operatorname{set}(P(M))=M^{\prime}$ and so, $\left|T_{P}^{n d}(M)\right|=1$.

Thus, for a definite $\mathcal{F}$-program $P$, the operator $T_{P}^{n d}$ is deterministic and, so, can be regarded as an operator with both the domain and codomain $\mathcal{P}(A t)$. We will write $T_{P}^{d}$, to denote it. Models, supported models and stable models of a definite monotone $\mathcal{F}$-program (for supported models we do not need the monotonicity assumption) can be introduced in terms of the operator $T_{P}^{d}$ in exactly the same way the corresponding concepts are defined in normal logic programming. In particular, the algebraic treatment of logic programming developed in (Fitting 2002 Przymusinski 1990, Denecker et al. 2000) applies to definite $\mathcal{F}$-programs and results in a natural and direct extension of normal logic programming. We note that this comment extends to 3- and 4-valued semantics of partial models, supported models and stable models (including the Kripke-Kleene semantics and the wellfounded semantics $)^{4}$.

We will explicitly mention just one result on definite monotone $\mathcal{F}$-programs (in fact, definite Horn programs) here, as it will be used later in the paper.

Proposition 10

Let $P$ be a definite Horn $\mathcal{F}$-program. Then $P$ has exactly one derivable model and this model is the least model of $P$.

Proof

Since $P$ is definite, it contains no constraint clauses and so, it has a model (Proposition 2). Thus, it has at least one $P$-computation. Let $\left(X_{n}\right)_{n=0,1, \ldots}$ and $\left(Y_{n}\right)_{n=0,1, \ldots}$ be two $P$-computations. By the definition, $X_{0}=\emptyset=Y_{0}$. Let us assume that for some $n \geq 0, X_{n}=Y_{n}$. By the definition of $P$-computations,

$$
X_{n+1} \in T_{P}^{n d}\left(X_{n}\right) \text { and } Y_{n+1} \in T_{P}^{n d}\left(Y_{n}\right) .
$$

By the induction hypothesis, $X_{n}=Y_{n}$. Thus, $T_{P}^{n d}\left(X_{n}\right)=T_{P}^{n d}\left(Y_{n}\right)$. Since $P$ is definite, $\left|T_{P}^{n d}\left(X_{n}\right)\right|=\left|T_{P}^{n d}\left(Y_{n}\right)\right|=1$ and so, $X_{n+1}=Y_{n+1}$. Thus, both computations coincide and $P$ has exactly one $P$-computation and so, exactly one derivable model. Since every model of $P$ contains a derivable model, it follows that the unique derivable model of $P$ is also a least model of $P$.

\footnotetext{
${ }^{4}$ Results in (Denecker et al. 2001 Pelov et al. 2004 Pelov. 2004) are related to this observation. They concern programs with aggregates, whose clauses have heads consisting of single atoms and so, are definite.
} 


\section{Encoding lparse programs as monotone $\mathcal{F}$-programs}

We will now investigate the relation between lparse programs (Niemelä et al. 1999. Simons et al. 2002) and programs with monotone abstract constraints. We start by reviewing the syntax and the semantics of lparse programs.

A weighted set of literals is a function $W: X \rightarrow\{\ldots,-1,0,1, \ldots\}$, where $X \subseteq$ $A t \cup\{\operatorname{not}(a): a \in A t\}$ is finite. We call $X$ the literal set of $W$ and denote it by $l \operatorname{set}(W)$. The set of atoms that appear in literals in $l \operatorname{set}(W)$ is the atom set of $W$. We denote it by aset $(W)$. Sometimes it will be convenient to write $W$ explicitly. To this end, we will write $W$ as

$$
\left\{a_{1}=w_{1}, \ldots, a_{m}=w_{m}, \operatorname{not}\left(b_{1}\right)=w_{1}^{\prime}, \ldots, \operatorname{not}\left(b_{n}\right)=w_{n}^{\prime}\right\},
$$

where the domain of the function $W$ is $\left\{a_{1}, \ldots, a_{m}, \operatorname{not}\left(b_{1}\right), \ldots, \operatorname{not}\left(b_{n}\right)\right\}$, and $w_{1}=W\left(a_{1}\right), \ldots, w_{n}^{\prime}=W\left(\operatorname{not}\left(b_{n}\right)\right)$, loosely following the lparse notation. Thus, when the domain of $W$ is $\{a, b, c\}$ and $W(a)=1, W(b)=2$, and $W(c)=1$, then we write $W$ as $\{a=1, b=2, c=1\}$.

An lparse atom (l-atom, for short) is an expression of the form $k W l$, where $W$ is a weighted set of literals, and $k$ and $l$ are integers such that $k \leq l$. By the literal set of an l-atom $A=k W l$ we mean $l \operatorname{set}(W)$ and write $l \operatorname{set}(A)$ to denote it (in a similar way, we extend the definition and the notation of the atoms set to the case of l-atoms).

We say that a set of atoms (interpretation) $M$ satisfies an l-atom $k W l$ if

$$
k \leq \sum_{\substack{p \in l \operatorname{set}(W) \\ p \in M}} W(p)+\sum_{\substack{\operatorname{not}(p) \in l \text { set }(W) \\ p \notin M}} W(p) \leq l
$$

( $M \models k W l$, in symbols). We note that it is easy to give an example of an inconsistent l-atom. For instance, $2\{a=1\} 2$ is inconsistent. We will use $I$ to denote any inconsistent constraint (it does not matter which, as all are equivalent to each other).

An lparse clause (l-clause, for short) is an expression $r$ of the form

$$
A \leftarrow B_{1}, \ldots, B_{n},
$$

where $A$ and $B_{i}, 1 \leq i \leq n$, are l-atoms. We call $A$ the head of $r$ and $\left\{B_{1}, \ldots, B_{n}\right\}$ the body of $r$. We denote them by $h d(r)$ and $b d(r)$, respectively. An lparse program is a finite set of l-clauses.

We say that a set $M \subseteq A t$ satisfies an l-clause $r$ if $M$ satisfies $h d(r)$ whenever it satisfies each l-atom in the body of $r$. We say that $M$ satisfies an lparse program $P$ if $M$ satisfies each l-clause in $P$. We write $M \models r$ and $M \models P$ in these cases, respectively.

We note that lparse programs allow both negative literals and negative weights in l-atoms. However, in (Simons et al. 2002) it is argued that negative weights can be expressed using negative literals and vice versa and, hence, one is inessential when the other is available. In fact, in (Simons et al. 2002) an l-atom with negative weights is treated simply as a shorthand for the corresponding constraint with nonnegative weights. We follow this approach here and from now on consider only l-atoms $k W l$, where $W$ assigns non-negative weights to literals. 
Before we continue, let us define $\mathcal{P} \mathcal{B}$ to be a set of all pseudo-boolean constraints of the form $P B(k, W, \leq)$, where $k$ is a non-negative integer and $W$ a weight function assigning to elements of $A t$ non-negative integers (cf. Section 2). Directly from the definition it follows that every constraint in $\mathcal{P B}$ is monotone.

Let us consider an l-atom $l W$ which contains no negative literals (and, as it is evident from the notation, no upper bound). In particular, $l \operatorname{set}(W)=\operatorname{aset}(W)$. Let $W^{\prime}$ be an extension of $W$, which assigns 0 to every atom $p \in A t \backslash \operatorname{aset}(W)$. We observe that a set $M \subseteq A t$ is a model of $l W$ if and only if $M$ is a model of the $\mathcal{P B}$-atom $A(X)$, where $A=P B\left(l, W^{\prime}, \leq\right)$ and $X=\operatorname{aset}(W)$. Therefore, we will regard such an l-atom $l W$ as a $\mathcal{P B}$-atom or, speaking more formally (but with some abuse of notation) we will denote by $l W$ the $\mathcal{P} \mathcal{B}$-atom $A(X)$.

If $W=\{a=1\}$ and $l=2$, then the corresponding $\mathcal{P} \mathcal{B}$-atom is inconsistent (it is one of many inconsistent $\mathcal{P} \mathcal{B}$-atoms). As in the case of l-atoms, we will write $I$ to denote (any) inconsistent $\mathcal{P} \mathcal{B}$-atom.

This discussion suggests that lparse programs built of l-atoms without negative literals and upper bounds can be viewed as Horn $\mathcal{P} \mathcal{B}$-programs. We will exploit that relationship below in the definition of the reduct, and will subsequently extend it to all lparse programs.

Let $P$ be an lparse program and let $M \subseteq A t$. An lparse-reduct of $P$ with respect to $M$ is a $\mathcal{P} \mathcal{B}$-program obtained by:

1. eliminating from $P$ every clause $r$ such that $M \not \forall B$, for at least one l-atom $B \in b d(r)$.

2. replacing each remaining l-clause $r=k W l \leftarrow k_{1} W_{1} l_{1}, \ldots, k_{n} W_{n} l_{n}$ with $\mathcal{P} \mathcal{B}$ clauses of the form

$$
1\{a=1\} \leftarrow k_{1}^{\prime} W_{1}^{\prime}, \ldots, k_{n}^{\prime} W_{n}^{\prime}
$$

where $a \in l \operatorname{set}(W) \cap M, W_{i}^{\prime}$ is $W_{i}$ restricted to $l \operatorname{set}\left(W_{i}\right) \cap A t$, and

$$
k_{i}^{\prime}=k_{i}-\sum_{\substack{\operatorname{not}(p) \in l \operatorname{set}\left(W_{i}\right) \\ p \notin M}} W_{i}(\operatorname{not}(p))
$$

(by our comments above, expressions of the form $l^{\prime} W^{\prime}$ denote $\mathcal{P} \mathcal{B}$-atoms). With some abuse of notation, we denote the reduct of $P$ with respect to $M$ by $P^{M}$ (the type of the program, an lparse program or a $\mathcal{P B}$-program, determines which reduct we have in mind). By our comments above, $P^{M}$ can be regarded as a definite Horn $\mathcal{P B}$-program. Thus, $P^{M}$ has a least model, $\operatorname{lm}\left(P^{M}\right)$ (Proposition 10). This model is the result of the canonical computation from $P^{M}$ with respect to $M$.

\section{Definition 9}

Let $P$ be an lparse program. A set $M \subseteq A t$ is an lparse-stable model of $P$ if $M=\operatorname{lm}\left(P^{M}\right)$ and $M \models P$.

We will now show that all lparse programs can be viewed as $\mathcal{P} \mathcal{B}$-programs. This task involves two steps. First, we show how to translate lparse programs to not-free lparse programs so that lparse-stable models are preserved. Second, we show that 
for not-free lparse programs the two definitions of stable models presented in the paper (Definitions 7 and 9) are equivalent.

An lparse program $P$ can be translated to a not-free lparse program $P^{\prime}$, as follows. We recall that by our earlier comments, we need to consider only lparse programs with no negative weights. For each negated literal $\operatorname{not}(b)$ appearing in $P$, introduce a new propositional atom $\bar{b}$ and an l-clause $\bar{b} \leftarrow 0\{b=1\} 0$. Then we replace each l-atom $k W l$ where the weighted set of literals $W$ is of the form (4) with an l-atom

$$
k\left\{a_{1}=w_{1}, \ldots, a_{m}=w_{m}, \overline{b_{1}}=w_{1}^{\prime}, \ldots, \overline{b_{n}}=w_{n}^{\prime}\right\} l .
$$

It is straightforward to show that this transformation preserves lparse-stable models in the following sense.

\section{Proposition 11}

Let $P$ be an lparse program, $P^{\prime}$ a not-free lparse program obtained by the translation above, and $B$ the set of new atoms introduced in the translation. Then,

- if $M$ is an lparse-stable model of $P$ then $M \cup\{\bar{b}: b \in B \backslash M\}$ is a lparse-stable model of $P^{\prime}$ and

- if $M^{\prime}$ is a lparse-stable model of $P^{\prime}$ then $M=M^{\prime} \backslash B$ is an lparse-stable model of $P$.

Now we show that not-free lparse programs can be translated to $\mathcal{P} \mathcal{B}$-programs. To simplify the description of the encoding and make it uniform, we assume that all bounds are present. Let $r$ be an l-clause

$$
k W l \leftarrow k_{1} W_{1} l_{1}, \ldots, k_{m} W_{m} l_{m} .
$$

We represent this l-clause by a pair of $\mathcal{P} \mathcal{B}$-clauses, $e_{1}(r)$ and $e_{2}(r)$ that we define as

$$
k W \leftarrow k_{1} W_{1}, \ldots, k_{m} W_{m}, \operatorname{not}\left(\left(l_{1}+1\right) W_{1}\right), \ldots, \operatorname{not}\left(\left(l_{m}+1\right) W_{m}\right),
$$

and

$$
I \leftarrow(l+1) W, k_{1} W_{1}, \ldots, k_{m} W_{m}, \operatorname{not}\left(\left(l_{1}+1\right) W_{1}\right), \ldots, \operatorname{not}\left(\left(l_{m}+1\right) W_{m}\right),
$$

respectively. We recall that the symbol $I$, appearing in the clause $e_{2}(r)$, stands for the inconsistent $\mathcal{P} \mathcal{B}$-atom introduced above.

Now, given a not-free lparse program $P$, we translate it into a $\mathcal{P B}$-program

$$
e(P)=\bigcup_{r \in P}\left\{e_{1}(r), e_{2}(r)\right\}
$$

Theorem 7

Let $P$ be a not-free lparse program. A set $M$ is an lparse-stable model of $P$ if and only if $M$ is a stable model of $e(P)$, as defined for $\mathcal{P B}$-programs. 
Proof

In the proof we will use the notation:

$$
P_{1}=\bigcup\left\{e_{1}(r): r \in P\right\} \text { and } P_{2}=\bigcup\left\{e_{2}(r): r \in P\right\} .
$$

Let us assume first that $M$ is an lparse-stable model of a not-free lparse program $P$. We will show that $M$ is a stable model of the $\mathcal{P} \mathcal{B}$-program $e(P)$, which in our terminology is equal to $P_{1} \cup P_{2}$.

Since $M$ is an lparse-stable model of $P$, it is a model of $P$ (Definition 9). Consequently, $M$ is a model of $P_{2}$. By Proposition 5 to complete this part of the proof, it suffices to show that $M$ is a stable model of the program $P_{1}$. To this end, we note that the definitions of the respective reducts imply that a clause

$$
1\{a=1\} \leftarrow k_{1} W_{1}, \ldots k_{m} W_{m}
$$

belongs to the lparse-reduct $P^{M}$ if and only if the reduct $P_{1}^{M}$ contains a clause

$$
k W \leftarrow k_{1} W_{1}, \ldots k_{m} W_{m}
$$

such that $a \in \operatorname{aset}(W)$ and $M \models k_{i} W_{i}$ for all $1 \leq i \leq m$.

From this relationship it follows that the results of the canonical computations from $P^{M}$ and $P_{1}^{M}$ with respect to $M$ coincide (we recall that both reducts are Horn $\mathcal{P B}$-programs). Since $M$ is the least model of $P^{M}$, it is the result of the canonical computation from $P^{M}$ with respect to $M$. Thus, $M$ is also the result of the canonical computation from $P_{1}^{M}$ with respect to $M$. In other words, $M$ is a derivable model of $P_{1}$ and, consequently, a stable model of $P_{1}$.

Conversely, let us assume that $M$ is a stable model of $P_{1} \cup P_{2}$. It follows that $M$ is a model of $P_{1} \cup P_{2}$ and, consequently, a model of $P$. Next, we note that since $M$ is a stable model of $P_{1} \cup P_{2}$, it is a stable model of $P_{1}$ (by Proposition 5). Thus, it is a derivable model of its reduct $P_{1}^{M}$ and, therefore, it is also the result of the canonical computation from $P_{1}^{M}$ with respect to $M$. Our observation about the relationship between the reducts $P_{1}^{M}$ of and $P^{M}$ holds now, as well. Consequently, $M$ is the result of the canonical computation from $P^{M}$ with respect to $M$. Thus, $M$ is a derivable model of $P^{M}$. Since $P^{M}$ is a definite Horn $\mathcal{P B}$-program, it has only one derivable model - its least model. It follows that $M$ is the least model of $P^{M}$ and, consequently, an lparse-stable model of $P$.

Theorem 7 shows that $\mathcal{P} \mathcal{B}$-programs can express arbitrary not-free lparse programs with only linear growth in the size of the program. The converse relationship holds, too: not-free lparse programs can represent arbitrary $\mathcal{P} \mathcal{B}$-programs without increasing the size of the representation. Let $r$ be a $\mathcal{P B}$-clause

$$
k W \leftarrow k_{1} W_{1}, \ldots, k_{m} W_{m}, \operatorname{not}\left(l_{1} V_{1}\right), \ldots, \operatorname{not}\left(l_{n} V_{n}\right)
$$

We define $f(r)$ as follows. If there is $i, 1 \leq i \leq n$, such that $l_{i}=0$, we set $f(r)=k W \leftarrow k W$ (in fact any tautology would do). Otherwise, we set

$$
f(r)=k W \leftarrow k_{1} W_{1}, \ldots, k_{m} W_{m}, 0 V_{1}\left(l_{1}-1\right), \ldots, 0 V_{n}\left(l_{n}-1\right) .
$$

Given a $\mathcal{P} \mathcal{B}$-program $P$, we define $f(P)=\{f(r): r \in P\}$. 
Theorem 8

Let $P$ be a $\mathcal{P B}$-program. A set of atoms $M$ is a stable model of $P$ (as defined for $\mathcal{P B}$-programs) if and only if $M$ is an lparse-stable model of $f(P)$.

Proof

First, we observe that $P$ and $f(P)$ have the same models. Next, similarly as before, we have that the lparse-reduct $[f(P)]^{M}$ contains a clause

$$
1\{a=1\} \leftarrow k_{1} W_{1}, \ldots k_{m} W_{m}, 0 V_{1}, \ldots, 0 V_{n}
$$

if and only if $P^{M}$ contains a clause of the form

$$
k W \leftarrow k_{1} W_{1}, \ldots k_{m} W_{m}
$$

such that $a \in \operatorname{aset}(W)$ and $M \models k_{i} W_{i}$ for all $1 \leq i \leq m$. Since in the clauses of the first type l-atoms $V_{i}$ are always true, as before, the results of the canonical computations from $P^{M}$ and $[f(P)]^{M}$ with respect to $M$ of $P$ coincide (we recall that both reducts are Horn $\mathcal{P} \mathcal{B}$-programs). Using this observation one can complete the proof by reasoning as in the previous proof.

Theorems [7 and 8 establish the equivalence of not-free lparse programs and $\mathcal{P} \mathcal{B}$ programs with respect to the stable model semantics. The translations $e$ and $f$ also preserve models. The equivalence between not-free lparse programs and $\mathcal{P} \mathcal{B}$ programs extends to supported models under the following concept of supportedness for lparse-programs.

\section{Definition 10}

Let $P$ be a not-free lparse program. A set of atoms $M$ is a supported model of $P$ if $M$ is a model of $P$ and if for every atom $a \in M$ there is an l-clause $A \leftarrow B_{1}, \ldots, B_{n}$ in $P$ such that $a \in \operatorname{aset}(A)$ and $M \models B_{i}, 1 \leq i \leq n$.

Indeed, we have the following two theorems (we only sketch a proof of one of them; the proof of the other one is similar).

Theorem 9

Let $P$ be a not-free lparse program. A set $M$ is an lparse-supported model of $P$ if and only if $M$ is a supported model of $e(P)$, as defined for $\mathcal{P} \mathcal{B}$-programs.

Proof

Let us denote $Q=e(P)$. Let $M$ be an lparse-supported model of $P$. We will show that $M$ is a supported model of $Q$. By our earlier observations, $P$ and $Q$ have the same models. Thus, $M$ is a model of $Q$. To complete the argument, we need to show that $M \subseteq h \operatorname{set}(Q(M))$. Let $a \in M$. Since $M$ is an lparse-supported model of $P$, there is an l-clause $r \in P$ such that $r=A \leftarrow B_{1}, \ldots, B_{n}, a \in \operatorname{aset}(A)$ and $M \models B_{i}$ for every $i, 1 \leq i \leq n$. It follows that $a \in \operatorname{aset}\left(h d\left(e_{1}(r)\right)\right)$ and that $M \models b d\left(e_{1}(r)\right)$. Since $e_{1}(r) \in Q, e_{1}(r) \in Q(M)$. Thus, $a \in h \operatorname{set}(Q(M))$. It follows that $M \subseteq h \operatorname{set}(Q(M))$ and so $M$ is a supported model of $Q$.

Conversely, let us assume that $M$ is a supported model of $Q$. Then $M$ is a model of $Q$ and so $M$ is a model of $P$, as well. Let $a \in M$. It follows that $a \in h \operatorname{set}(Q(M))$. 
Since each clause of the form $e_{2}(r)(r \in P)$ is a constraint, there is an l-clause $r \in P$ such that clause $e_{1}(r) \in Q$ such that $M \models b d\left(e_{1}(r)\right)$ and $a \in h \operatorname{set}\left(h d\left(e_{1}(r)\right)\right)$. Let $r=A \leftarrow B_{1}, \ldots, B_{n}$. It follows that $a \in \operatorname{aset}(A)$ and that $M \models B_{i}, 1 \leq i \leq n$. Thus, $M$ is an lparse-supported model of $P$.

\section{Theorem 10}

Let $P$ be a $\mathcal{P B}$-program. A set of atoms $M$ is a stable model of $P$ (as defined for $\mathcal{P B}$-programs) if and only if $M$ is an lparse-stable model of $f(P)$.

It follows from the results in this section that the translations $e$ and $f$ uniformly preserve basic semantics of not-free lparse and $\mathcal{P} \mathcal{B}$-programs, and allow us to view not-free lparse programs as $\mathcal{P} \mathcal{B}$-programs and vice versa.

We also note that this equivalence demonstrates that lparse programs with the semantics of stable models as defined in (Niemelä et al. 1999) can be viewed as a generalization of normal logic programming. It follows from Theorems [6] and 8 that the encoding of normal logic programs as lparse programs, defined as the composition of the translation $P \mapsto P^{p b}$ described in Section 8 (we note that the constraint $P B$ belongs to the class $\mathcal{P B}$ ) and the translation $f$, preserves the semantics of models, supported models and stable models (an alternative proof of this fact, restricted to the case of stable models was first given in (Simons et al. 2002) and served as a motivation for the class of lparse programs and its stable-model semantics). This result is important, as it is not at all evident that the reduct used in (Simons et al. 2002), leads to fixpoints that generalize the semantics of stable models as defined in (Gelfond and Lifschitz 1988).

Given that the formalisms of not-free lparse and $\mathcal{P} \mathcal{B}$-programs are equivalent, it is important to stress what differentiates them. The advantage of the formalism of not-free lparse programs is that it does not require the negation operator in the language. The strength of the formalism of $\mathcal{P B}$-programs lies in the fact that its syntax so closely resembles that of normal logic programs, and that the development of the theory of $\mathcal{P B}$-programs so closely follows that of the normal logic programming.

\section{Monotone $\mathcal{F}$-programs and disjunctive logic programs}

(Sakama and Inoue 1994) introduced and investigated a semantics of possible models of disjunctive logic programs. It turns out that this semantics is different from the semantics proposed by Minker (Minker 1982) and from that of Gelfond and Lifschitz (Gelfond and Lifschitz 1991 Przymusinski 1991). In this section, we will show that the formalism of monotone $\mathcal{F}$-programs generalizes the semantics of possible models. For the purpose of our discussion, we will extend the use of the

terms head, body, $M$-applicability, and notation $P^{M}, h d(r), b d(r)$ to the case of disjunctive programs.

Definition 11

(Sakama and Inoue 1994) Let $P$ be a disjunctive logic program. A split program for $P$ is any normal logic program that can be obtained by the following procedure. 
First, we select for each clause $r$ in $P$, a set $S_{r} \subseteq h d(r)$. Next, we replace $r$ with clauses of the form $A \leftarrow \operatorname{body}$, where $A \in S_{r}$, if $S_{r} \neq \emptyset$, and with the (constraint) clause $\leftarrow b d(r)$, if $S_{r}=\emptyset$.

A set $M$ of atoms is a possible model of $P$ if $M$ is a stable model (in the sense of Gelfond and Lifschitz (Gelfond and Lifschitz 1988)) of a split program for $P$.

We point out that we allow for disjunctive clauses to have empty heads (that is, we allow constraint clauses). We also allow that sets $S_{r}$ be empty. Consequently, split programs may contain constraints even if the original program does not.

\section{Example 6}

Let $P$ be the disjunctive program:

$$
P=\{a \vee b \vee c \leftarrow, \quad a \vee c \leftarrow \operatorname{not}(b), \quad b \leftarrow \operatorname{not}(c), \quad c \leftarrow \operatorname{not}(a)\}
$$

Then, the program $Q$ :

$$
Q=\{a \leftarrow, \quad a \leftarrow \operatorname{not}(b), \quad c \leftarrow \operatorname{not}(b), \quad b \leftarrow \operatorname{not}(c), \quad \leftarrow \operatorname{not}(a)\} .
$$

is an example of a split program for $P$ (given by the following subsets of the sets of atoms in the heads of the clauses: $\{a\},\{a, c\},\{b\}$ and $\emptyset$, respectively). Moreover, since $\{a, b\}$ is a stable model of $Q,\{a, b\}$ is a possible model of $P$.

If $M$ is a model of a disjunctive program $P$, by $s(P, M)$ we denote the split program of $P$ determined by sets $S_{r}=M \cap h d(r)$. We have the following simple characterization of possible models.

\section{Proposition 12}

Let $P$ be a disjunctive program. A set of atoms $M$ is a possible model of $P$ if and only if $M$ is a stable model of the split program $s(P, M)$.

\section{Proof}

If $M$ is a possible model of $P$, then $M$ is a stable model of a split program of $P$, say $Q$. Let us assume that $Q$ is determined by sets $S_{r} \subseteq h d(r)$, where $r \in P$.

For every clause $r \in P$, if $r$ is $M$-applicable then all clauses it contributes to $Q$ are $M$-applicable, too, as they have the same body as $r$. Since $M$ is a model of $Q$ (being a stable model of $Q$ ), we obtain that $S_{r} \subseteq M$. Thus, $Q(M) \subseteq s(P, M)$.

Directly from the definition of $s(P, M)$ we obtain that $M$ is a model of $s(P, M)$. Thus, $M$ is a model of $[s(P, M)]^{M}$ and, consequently, $\operatorname{lm}\left([s(P, M)]^{M}\right)$ exists. Moreover, it follows that $\operatorname{lm}\left([s(P, M)]^{M}\right) \subseteq M$ (indeed, all non-constraint clauses in $s(P, M)$ have heads from $M)$.

Since $M$ is a stable model of $Q, M=\operatorname{lm}\left(Q^{M}\right)$. Thus, it follows that $M=$ $\operatorname{lm}\left([Q(M)]^{M}\right)$ and so, we obtain:

$$
M=\operatorname{lm}\left([Q(M)]^{M}\right) \subseteq \operatorname{lm}\left([s(P, M)]^{M}\right) \subseteq M .
$$

Thus, $\operatorname{lm}\left([s(P, M)]^{M}\right)=M$ or, in other words, $M$ is a stable model of $s(P, M)$. The converse implication follows by the definition. 
Let $r$ be a disjunctive logic program clause of the form:

$$
c_{1} \vee \ldots \vee c_{k} \leftarrow a_{1}, \ldots, a_{m}, \operatorname{not}\left(b_{1}\right), \ldots, \operatorname{not}\left(b_{n}\right)
$$

where all $a_{i}, b_{i}$ and $c_{i}$ are atoms. We encode this clause as a program clause with cardinality atoms:

$$
r^{c a}=1\left\{c_{1}, \ldots, c_{k}\right\} \leftarrow 1\left\{a_{1}\right\}, \ldots, 1\left\{a_{m}\right\}, \operatorname{not}\left(1\left\{b_{1}\right\}\right), \ldots, \operatorname{not}\left(1\left\{b_{n}\right\}\right)
$$

(If all $a_{i}$ and $b_{i}$ are distinct, the following translation could be used instead: $1\left\{c_{1}, \ldots, c_{k}\right\} \leftarrow m\left\{a_{1}, \ldots, a_{m}\right\}, \operatorname{not}\left(1\left\{b_{1}, \ldots, b_{n}\right\}\right)$.) We note that if $k=0$, that is, the head of $r$ is empty, the rule $r^{c a}$ has the constraint $1 \emptyset$ in the head, which is inconsistent. In this case, $r^{c a}$ is a constraint clause.

For a disjunctive logic program $P$, we define $P^{c a}=\left\{r^{c a}: r \in P\right\}$ (ca in the subscript stands for "cardinality atoms"). Since cardinality constraints are monotone, the concept of a stable model of the program $P^{c a}$ is well defined. We have the following theorem.

\section{Theorem 11}

Let $P$ be a disjunctive logic program. A set of atoms $M$ is a possible model of $P$ if and only if $M$ is a stable model of the program $P^{c a}$ (in the sense, we defined in this paper).

\section{Proof}

We first note that $[s(P, M)]^{M}=s\left(P^{M}, M\right)$. Thus, by Proposition 12 it follows that $M$ is a possible model of $P$ if and only if $M$ is a least model of $s\left(P^{M}, M\right)$. We also note that $\left[P^{c a}\right]^{M}=\left[P^{M}\right]^{c a}$. Thus, $M$ is a stable model of $P^{c a}$ if and only if $M$ is a derivable model of $\left[P^{M}\right]^{c a}$.

It follows that in order to prove the assertion it suffices to show that for every positive (no negation in the bodies of clauses) disjunctive program $P, M$ is a least model of $s(P, M)$ if and only if $M$ is a derivable model of $P^{c a}$. We will now prove this claim. To simplify notation, we will write $Q$ instead of $P^{c a}$.

First, we note $P$ and $Q$ have the same models. Thus, each side of the equivalence implies that $M$ is a model of $Q$. In particular, it follows (no matter which implication we are proving) that $Q$ has a canonical computation $t^{Q, M}=\left(X_{n}^{Q, M}\right)_{n=0,1, \ldots}$. Next, we observe that for every $X \subseteq M$, the definitions of $Q$ and $s(P, M)$ imply that

$$
h \operatorname{set}(Q(X)) \cap M=h d(s(P, M)(X))=T_{s(P, M)}(X) .
$$

In particular, since $X_{n+1}^{Q, M}=h \operatorname{set}\left(Q\left(X_{n}^{Q, M}\right)\right) \cap M$, for every $n=0,1, \ldots$, we have

$$
X_{n+1}^{Q, M}=T_{s(P, M)}\left(X_{n}^{Q, M}\right)
$$

These identities imply that the result of the canonical $Q$-computation for $M$ and the least model of $s(P, M)$ coincide. Consequently, $M$ is a derivable model of $Q$ if and only if $M$ is a least model of $s(P, M)$ as claimed. 


\section{Discussion}

In the paper, we introduced and studied the formalism of $\mathcal{F}$-programs. When all constraints in $\mathcal{F}$ are monotone, this formalism offers an abstract framework for integrating constraints into logic programming. It exploits and builds on analogies with normal logic programming. Most concepts and techniques for monotone $\mathcal{F}$ programs are closely patterned after their counterparts developed there and so, normal logic programming can be viewed as a fragment of the theory of monotone $\mathcal{F}$-programs. Importantly, the same is the case for other nonmonotonic systems namely, the disjunctive logic programming with the possible-model semantics of (Sakama and Inoue 1994), and for the formalism of logic programs with weight constraints (Simons et al. 2002). For these two formalisms, monotone $\mathcal{F}$-programs help to explain the nature of their relationship with normal logic programming, hidden by their original definitions.

In this paper, we developed a sound foundation for the theory of monotone $\mathcal{F}$ programs. Recently, the theory of monotone $\mathcal{F}$-programs was developed further. (Liu and Truszczyński 2005b) demonstrated that Fages lemma (Fages 1994), and the concepts of the program completion and a loop formula extend to the setting of monotone $\mathcal{F}$-programs. The latter two properties allow one to reduce stable-model computation for programs with weight constraints to the problem of computing models of propositional theories extended with weight atoms (referred to as pseudoboolean constraints in the satisfiability community). (Liu and Truszczyński 2005a) exploited this reduction and developed an algorithm to compute stable models of programs with weight constraints by using off-the-shelf solvers of pseudo-boolean constraints such as those described in (Barth 1995, Walser 1997, Aloul et al. 2002, Liu and Truszczyński 2003 East and Truszczyński 2004).

There are strong analogies between the approach we propose and develop here and some of the techniques discussed in (Sakama and Inoue 1994) in the context of disjunctive programs with the semantics of possible models. One way to look at the results of our paper is that it extends the way (Sakama and Inoue 1994) handles nondeterminism, inherent in disjunctive logic programs, to the abstract setting of monotone $\mathcal{F}$-programs. In particular, (Sakama and Inoue 1994) presents a computational procedure for disjunctive programs without negation, which can be shown to be closely related to our notion of a $P$-computation. That paper also introduces a nondeterministic provability operator, defined for positive disjunctive programs. Three aspects differentiate our work from (Sakama and Inoue 1994). Most importantly, we study here a much broader class of programs than disjunctive ones. Secondly, we define a provability operator on the class of all monotone $\mathcal{F}$-programs and not just positive ones. Finally, we consistently exploit properties of this operator, and align our approach with the standard operator-based development of normal logic programming (Apt 1990 Fitting 2002).

The emergence of a nondeterministic one-step provability operator warrants additional comments. Nondeterministic provability operators were considered before in the context of logic programming. We already noted that (Sakama and Inoue 1994) proposed a provability operator similar to the one we introduced here (although only 
for the class of positive disjunctive programs). (Sakama and Inoue 1995) proposed another operator designed to capture a different computational process arising in the context of paraconsistent systems. Finally, (Pelov and Truszczynski. 2004) presented a characterization of answer sets of disjunctive logic programs in terms of yet another nondeterministic provability operator. However, the operator we proposed here exhibits the closest parallels with the van Emden-Kowalski operator and opens up a possibility of generalizing the approximation theory proposed in (Denecker et al. 2000) to the case of monotone $\mathcal{F}$-programs. However, for that to happen, one will need techniques for handling nondeterministic operators on lattices, similar to those presented for the deterministic operators in (Denecker et al. 2000, Denecker et al. 2004). Developing such techniques is an open problem.

\section{Acknowledgments}

The authors wish to thank the anonymous reviewers for their detailed comments and suggestions, which helped improve the paper. The second author was supported by the Academy of Finland grant 211025. The other two authors were supported by the NSF grants IIS-0097278 and IIS-0325063.

\section{References}

Aloul, F., Ramani, A., Markov, I., And Sakallah, K. 2002. PBS: a backtracksearch pseudo-boolean solver and optimizer. In Proceedings of the 5th International Symposium on Theory and Applications of Satisfiability, (SAT-02). 346 - 353.

Apt, K. 1990. Logic programming. In Handbook of theoretical computer science, J. van Leeuven, Ed. Elsevier, 493-574.

BABOvich, Y. AND Lifschitz, V. $2002 . \quad$ Cmodels package. http://www.cs.utexas.edu/users/tag/cmodels.html

BARAL, C. 2003. Knowledge representation, reasoning and declarative problem solving. Cambridge University Press.

BARTh, P. 1995. A Davis-Putnam based elimination algorithm for linear pseudo-boolean optimization. Tech. Rep., Max-Planck-Institut für Informatik. MPI-I-95-2-003.

Calimeri, F., Faber, W., Leone, N., and Perri, S. Declarative and Computational Properties of Logic Programs with Aggregates. In Proceedings of the 19th International Joint Conference on Artificial Intelligence (IJCAI-05), 406-411.

Clark, K. 1978. Negation as failure. In Logic and data bases, H. Gallaire and J. Minker, Eds. Plenum Press, 293-322.

Dell'Armi, T., Faber, W., Ielpa, G., Leone, N., And Pfeifer, G. 2003. Aggregate functions in disjunctive logic programming: semantics, complexity, and implementation in DLV. In Proceedings of the 18th International Joint Conference on Artificial Intelligence (IJCAI-2003). Morgan Kaufmann, 847-852.

Denecker, M., Marek, V., And Truszczyński, M. 2000. Approximations, stable operators, well-founded fixpoints and applications in nonmonotonic reasoning. In Logic-Based Artificial Intelligence, J. Minker, Ed. Kluwer Academic Publishers, 127-144.

Denecker, M., Marek, V., And Truszczyński, M. 2004. Ultimate approximation and its application in nonmonotonic knowledge representation systems. Information and Computation 192, 84-121. 
Denecker, M., Pelov, N., And Bruynooghe, M. 2001. Ultimate well-founded and stable semantics for logic programs with aggregates. In Logic Programming, Proceedings of the 2001 International Conference on Logic Programming (ICLP-01). LNCS 2237. Springer, 212-226.

EAst, D. And Truszczyński, M. 2006. Predicate-calculus based logics for modeling and solving search problems. ACM Transactions on Computational Logic 7, 38-83.

Faber, W., Leone, N., And Pfeifer, G. 2004. Recursive aggregates in disjunctive logic programs: Semantics and complexity. In Proceedings of the 9th European Conference on Artificial Intelligence (JELIA-04). LNAI 3229. Springer, $200-212$.

FAGES, F. 1994. Consistency of Clark's completion and existence of stable models. Journal of Methods of Logic in Computer Science 1, 51-60.

FitTing, M. C. 2002. Fixpoint semantics for logic programming - a survey. Theoretical Computer Science 278, 25-51.

Gelfond, M. And LeOne, N. 2002. Logic programming and knowledge representation the A-prolog perspective. Artificial Intelligence 138, 3-38.

Gelfond, M. And Lifschitz, V. 1988. The stable semantics for logic programs. In Proceedings of the 5th International Conference on Logic Programming (ICLP-88). MIT Press, 1070-1080.

Gelfond, M. And Lifschitz, V. 1991. Classical negation in logic programs and disjunctive databases, New Generation Computing 9, 365-385.

Leone, N., Pfeifer, G., Faber, W., Eiter, T., Gottlob, G., Perri, S., And ScArCEllo, F. 2006. The dlv system for knowledge representation and reasoning. ACM Transactions on Computational Logic. To appear, available at http://xxx.lanl.gov/abs/cs.AI/0211004

Lifschitz, V. 1996. Foundations of logic programming. In Principles of Knowledge Representation, 69-127. CSLI Publications.

Lin, F. AND ZhaO, Y. 2002. ASSAT: Computing answer sets of a logic program by SAT solvers. In Proceedings of the 18th National Conference on Artificial Intelligence (AAAI-02). AAAI Press, 112-117.

LiU, L. AND TRuszczyński, M. 2003. Local-search techniques in propositional logic extended with cardinality atoms. In Proceedings of the 9th International Conference on Principles and Practice of Constraint Programming (CP-2003). LNCS 2833. Springer, 495-509.

Liu, L. And Truszczyński, M. 2005a. Pbmodels - software to compute stable models by pseudoboolean solvers. In Logic Programming and Nonmonotonic Reasoning, Proceedings of the 8th International Conference (LPNMR-05). LNAI 3662. Springer, 410-415.

LiU, L. AND Truszczyński, M. 2005b. Properties of programs with monotone and convex constraints. In Proceedings of the 20th National Conference on Artificial Intelligence (AAAI-05). AAAI Press, 701-706.

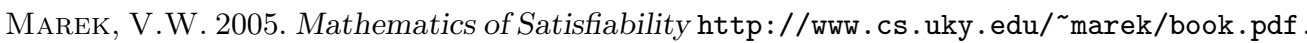

MareK, V., Niemelä, And TruszczyŃski, M. 2004. Characterizing stable models of logic programs with cardinality constraints. In Logic Programming and Nonmonotonic Reasoning, Proceedings of the 7th International Conference (LPNMR-04), LNAI 2923. Springer, 154-166.

MareK, V.W. And Remmel, J.B. 2004. Set Constraints in Logic Programming. In Logic Programming and Nonmonotonic Reasoning, Proceedings of the 7th International Conference (LPNMR-04). LNAI 2923. Springer, 154-167.

MAREK, V. AND TRUszczyńsKi, M. 2004. Logic programs with abstract constraint atoms. In Proceedings of the 19th National Conference on Artificial Intelligence (AAAI-04). AAAI Press, 86-91. 
MinkeR, J. 1982. On indefinite databases and the closed world assumption. In Proceedings of the 6th conference on automated deduction. LNCS 138. Springer, 292-308.

Niemelä, I. And Simons, P. 1997. Smodels - an implementation of the stable model and well-founded semantics for normal logic programs. In Logic Programming and Nonmonotonic Reasoning, Proceedings of the 4th International Conference (LPNMR97). LNAI 1265. Springer, 420-429.

Niemelä, I., Simons, P., And Soininen, T. 1999. Stable model semantics of weight constraint rules. In Logic Programming and Nonmonotonic Reasoning, Proceedings of the 5th International Conference (LPNMR-99). LNAI 1730. Springer, 317-331.

Pelov., N. 2004. Semantics of logic programs with aggregates. PhD Dissertation. Department of Computer Science, K.U.Leuven, Leuven, Belgium.

Pelov, N., Denecker, M., And Bruynooghe, M. 2004. Partial stable models for logic programs with aggregates. In Logic Programming and Nonmonotonic Reasoning, Proceedings of the 7th International Conference (LPNMR-04), LNAI 2923. Springer, 207-219.

Pelov, N. And Truszczynski, M. 2004. Semantics of disjunctive programs with monotone aggregates - an operator-based approach. In Proceedings of the 10th International Workshop on Non-Monotonic Reasoning (NMR-04). 327-334.

PrZymusinski, T. 1990. The well-founded semantics coincides with the three-valued stable semantics. Fundamenta Informaticae 13(4), 445-464.

PrZymusinski, T. 1991. Stable semantics for disjunctive programs, New Generation Computing 9, 401-424.

Sakama, C. And Inoue, K. 1994. An alternative approach to the semantics of disjunctive logic programs and deductive databases. Journal of Automated Reasoning 13, 145-172.

Sakama, C. And Inoue, K. 1995. Paraconsistent Stable Semantics for Extended Disjunctive Programs. Journal of Logic and Computation 5, 265-285.

Simons, P., Niemelä, I., And Soininen, T. 2002. Extending and implementing the stable model semantics. Artificial Intelligence 138, 181-234.

Son, C., Pontelli, E., AND Tu, P.H. 2006. Answer sets for logic programs with arbitrary abstract constraint atoms. In Proceedings of the 21st National Conference on Artificial Intelligence (AAAI-06). AAAI Press, 129-134.

VAN EMdEN, M. AND KowALSKI, R. 1976. The semantics of predicate logic as a programming language. Journal of the ACM 23, 4, 733-742.

WALSER, J. 1997. Solving linear pseudo-boolean constraints with local search. In Proceedings of the 14th National Conference on Artificial Intelligence (AAAI-97). AAAI Press, 269-274. 